\title{
Model refactoring by example: A multi-objective search based software engineering approach
}

\author{
Adnane Ghannem $^{1}$ (D) | Marouane Kessentini ${ }^{2}$ | Mohammad Salah Hamdi ${ }^{1}$ (D) | \\ Ghizlane El Boussaidi ${ }^{3}$
}

${ }^{1}$ Information Systems Department, Ahmed Bin Mohammed Military College, Qatar

${ }^{2}$ Computer and Information Science Department, University of Michigan, USA

${ }^{3}$ Software Engineering and IT Department, École de Technologie Supérieure, Canada Correspondence

Adnane Ghannem, Information Systems Department, Ahmed Bin Mohammed Military College, Qatar.

Email: adnane.ghannem@abmmc.edu.qa

Funding information

NSF, Grant/Award Number: 1661422; NPRP, Grant/Award Number: 7-662-2-247; Qatar

Foundation; Qatar Research Fund

\begin{abstract}
Declarative rules are frequently used in model refactoring in order to detect refactoring opportunities and to apply the appropriate ones. However, a large number of rules is required to obtain a complete specification of refactoring opportunities. Companies usually have accumulated examples of refactorings from past maintenance experiences. Based on these observations, we consider the model refactoring problem as a multi objective problem by suggesting refactoring sequences that aim to maximize both structural and textual similarity between a given model (the model to be refactored) and a set of poorly designed models in the base of examples (models that have undergone some refactorings) and minimize the structural similarity between a given model and a set of well-designed models in the base of examples (models that do not need any refactoring). To this end, we use the Non-dominated Sorting Genetic Algorithm (NSGA-II) to find a set of representative Pareto optimal solutions that present the best trade-off between structural and textual similarities of models. The validation results, based on 8 real world models taken from open-source projects, confirm the effectiveness of our approach, yielding refactoring recommendations with an average correctness of over $80 \%$. In addition, our approach outperforms 5 of the state-of-the-art refactoring approaches.
\end{abstract}

\section{KEYWORDS}

model evolution, NSGA-II, Pareto front, refactoring by example, software maintenance

\section{I INTRODUCTION}

Maintenance practice consists of modifying existing software while preserving its integrity. ${ }^{1}$ These modifications are incremental and aim to update some functionality or correct some design flaws and fix some bugs. These software maintenance activities become more complex when the size of the system and the number of requirements increase over time. ${ }^{2}$ Therefore, it is important to provide automated and semi-automated software maintenance tools to improve the quality of software systems.

Software developers are, in general, interested to restructure the software system to improve its structure and design before a new release. This restructuring process is called refactoring. ${ }^{3}$ Fowler $^{2}$ defined the refactoring as the process that improves the software structure while preserving its external behavior. The refactoring strategy involves several activities ${ }^{3}$ including the detection of refactoring opportunities in a given software and the recommendation of which refactorings to apply. Many researchers have been working on providing support for refactoring $\left(\mathrm{eg},{ }^{2,4,5}\right)$ focusing on the code level (eg, code smells $\left.{ }^{6}\right)$. Few approaches addressed detecting and recommending refactorings at the model level $\left(\mathrm{eg}^{7-10}\right)$. However, model-driven engineering (MDE) is a promising approach that manages software systems' complexity and specifies domain concepts effectively. ${ }^{11} \mathrm{MDE}$ considers the models as primary artifacts in the software systems. It consists of refining and successively transforming abstract models into more concrete models including executable source code. In this context, refactoring is a specific type of model transformation that aims at improving the quality of a given model; for example by improving the design of an existing design model by applying a design pattern which can be encoded as a model transformation. ${ }^{12}$

The rise of MDE increased the interest and the needs for tools supporting refactoring at the model-level. However, many challenges need to be overcome when building such a tool. Some of these challenges were identified in Mens et al, ${ }^{8}$ and they include issues related to assessing model 
quality, ensuring synchronization, and coherence between models (including source code), preserving behavior, etc. Most of existing refactoring tools offer a semi-automatic support ${ }^{8}$ because part of the necessary knowledge for performing the refactoring remains implicit in designers' expertise and programming behavior. Thus, recognizing model refactoring opportunities remains a big challenge that is related to the model marking process within MDE context which requires design knowledge and expertise. ${ }^{12}$ Furthermore, declarative rules represent a common technique in most of existing work on refactoring in order to detect and correct defects (ie, refactoring opportunities) and defect types can be very important. ${ }^{13}$ Another common issue to most of refactoring approaches is the problem of sequencing and composing refactoring rules that is, generally, related to the control of rules' applications within a rule-based transformational approach.

To overcome some of these issues, many refactoring approaches are using a search-based approach where the refactoring is considered as an optimization problem $\left(\mathrm{eg},{ }^{14-19}\right)$. Search-based refactoring approaches adapted some of the known heuristics methods such as simulated annealing and Hill_climbing as proposed in O' Keeffe and O' Cinneide ${ }^{14}$ and Seng et $\mathrm{al}^{15}$ and genetic algorithms (GAs) as proposed in Kessentini et al. ${ }^{16}$ In previous work, ${ }^{17}$ we proposed a by example approach that suggests refactorings to correct models. The approach uses single-objective optimization to find the best refactorings sequences that maximize the structural similarity between the model under analysis and a set of model refactoring examples. We calculated the structural similarity based on a set of metrics. Other optimization goals were considered in search-based refactoring approaches (eg, reducing the refactoring effort, ${ }^{18}$ improving the software structure ${ }^{15}$ ). Harman et al ${ }^{19}$ have proposed a multi-objective approach that uses 2 software metrics (CBO: coupling between objects, and SDMPC: standard deviation of methods per class) to define 2 optimization objectives. Most of these approaches relied on the structural information (ie, a combination of software metrics) to formulate their fitness functions and do not consider the design consistency in the optimization process. However, to suggest meaningful refactorings and to reduce the number of possible refactorings, both quality and consistency of the model to be refactored should be considered.

This paper represents an extension of our previous work ${ }^{20}$ having as goal to propose a new multi-objective optimization approach that aims at finding the best sequence of refactorings that optimizes 2 objectives: (1) the first objective consists of maximizing both the structural and the textual similarity between a given model (ie, the model to be refactored) and a set of models in the base of examples (ie, models that have undergone some refactorings); and (2) the second objective consists of minimizing the structural similarity between a given model (ie, the model to be refactored) and a set of well-designed models in the base of examples (ie, models that do not need any refactoring). We start from the observation that required knowledge to propose suitable refactorings for a given new object-oriented model may be inferred from other similar existing models' refactorings. We defined 2 levels of textual and structural similarities (measures) between these models and the given model. To combine these measures, we adapted the Non-dominated Sorting Genetic Algorithm (NSGA-II) ${ }^{21}$ that aims at finding a set of representative Pareto optimal solutions in a single run. Our approach takes as input an initial model to refactor, a base of examples of models and their subsequent refactorings, and a list of structural metrics and textual measures. It generates as output a solution as a sequence of refactorings that should be applied to the initial model. The process of generating this solution is the mechanism that finds a sequence of refactoring operations that represent the best trade-off between the 2 criteria of structural and textual similarities between the model to refactor and good/bad design examples.

The intuition behind this work is that we can use data collected from previous releases or projects within the same organization. The "good" and "bad" examples are defined based on the developers' design and programming practices. In our experiments, we showed that open source systems could represent a very good starting point, but to get optimal results it is always better that the developers/users provide a good and bad set of examples from their previous projects to get optimal results. These examples could be found, for example, in the review reports where some bad examples in terms of quality are described. Another option is to use a set of quality metrics to define what is considered as good or bad by the developers to extract a set of examples.

The approach could be considered as a data-mining technique because it is based on the exploration of design examples to identify relevant refactorings for a new context (project). We believe that one of the main key limits is related to the automated adaptation of refactorings applied in a similar context to a new one. In some situations, a user interaction is required and not all the refactorings that are recommended can be applied. However, the problem is a bit easier at the model level comparing to the code level. In our approach, we do not try to extract association rules like classical data mining techniques. However, the multi-objective approach adapts the refactoring solution as much as possible to an existing example while considering the applicability of the refactorings. Thus, we do not generate "templates" from the refactoring examples then apply them, but our goal is to "optimize" as much as possible the similarity between the examples and the identified model fragment to be refactored.

The main contributions of the paper can be summarized as follows:

- We introduce a novel by example multi-objective refactoring approach taking into consideration the design consistency when comparing between the model to be refactored and existing model examples to suggest refactoring solutions.

- We report the result of an empirical study of NSGA-II $t$ applied to 8 open source systems. We compare the results of our approach to those obtained in 6 other approaches (a mono objective approach (MONO), MOREX, ${ }^{17}$ random search, multi-objective particle swarm optimization (MOPSO), ${ }^{21}$ a genetic programming approach GP, ${ }^{22}$ and our previous work that is not based on the use of good design examples ${ }^{20}$ ). The result of this comparison provides evidence to support the claim that our proposal enables the generation of refactoring solutions with a high precision.

The goal of our experimentation is to find out whether our approach could propose meaningful sequences of refactorings to correct design defects within models (eg, class diagrams). Indeed, our approach addresses 2 research questions: 
- RQ1: To what extent can the proposed approach generate correct sequences of refactorings?

- RQ2: To what extent can the design consistency aspect improve the efficiency of our proposal to generate meaningful refactoring solutions?

To answer RQ1, we assessed the precision and recall of our approach on 8 open source projects. To answer RQ2, we compared our results to those produced by our previous work called MOREX, ${ }^{17}$ MOREX, ${ }^{17} \mathrm{GP},{ }^{22}$ MOPSO, ${ }^{21}$ and NSGA-Il without the use of good design examples. ${ }^{20}$

The rest of this paper is organized as follows. Section 2 presents the overall approach and the details of our adaptation of the multi-objective evolutionary algorithm NSGA-II to the model refactoring problem. Section 3 describes the supporting tools and experimental settings and presents results and discussion. Section 4 discussed the related works. Finally, we conclude and outline some future directions to our work in Section 5 .

\section{I MODEL REFACTORING USING MULTI OBJECTIVE OPTIMIZATION}

\section{1 | Approach overview}

The proposed approach exploits model refactorings' examples and NSGA- $\|^{23}$ to automatically suggest (to the user) refactorings' sequences possibly applicable on a given model. The user is responsible for applying these refactorings. For example, for a rename refactoring, our approach does not decide for the new name, but it suggests the mentioned refactoring operation, and we give hand to the user to choose the right name based on his knowledge. The general structure of our approach is introduced in Figure 1. It takes as input a set of bad designed models (label A) (ie, existing models and their related refactorings), a set of well-designed models that do not need refactorings (label B), an initial model (label C), and takes as controlling parameters a set of software metrics (label D). The approach generates as output a sequence of refactorings that can be applied to the initial model. The goal of our approach is to recommend the refactorings that make the design similar to good ones in terms of quality; thus, we implicitly considered the fact that the refactorings should improve the quality by meeting quality values similar the ones of the good design. The generation process of refactorings' sequences (Figure 1) can be seen as the mechanism that finds the best way to combine refactoring operations among the ones in the base of examples, in such a way to (1) maximize the structural and the textual similarities between entities to be refactored in the initial model and entities of the models (bad designed models) and (2) minimize the structural similarity between entities to be refactored in the initial model and entities of the models (well-designed systems). The structural similarity between 2 entities (eg, classes) is computed using software metrics of these entities while their textual similarity is computed using textual measures based on WordNet. ${ }^{24}$

In the proposed approach, we considered a subset of the 72 refactorings defined in Fowler ${ }^{2}$ possibly applicable to UML class diagrams. For example, "Rename_Method", "Move_Method", "Move_Attribute", "Extract_Class", etc. may be applied on design models, while "Inline_Method", "Extract_Method", "Replace_Temp_With_Query", etc. Table 1 shows the list of 12 refactorings considered in our approach chosen based on 2 factors: (1) they can be applied at the class diagram level; and (2) they can be linked to a set of metrics (ie, metrics that are impacted when applying these refactorings).

Table 2 shows the list of 16 metrics used in our approach and that could be applied to class diagrams (eg, NA, NMeth, NDep, etc.). These metrics include the 11 metrics defined in Genero et $\mathrm{al}^{25}$ to which we have added a set of simple metrics (eg, NPvMeth, NPbMeth). All these metrics are related to the class entity, which is the main entity in a class diagram. These metrics are used to compute the structural similarities between classes from the initial model and those in the base of examples. We believe that the textual similarity measure, described in the next section, considers the consistency between the elements.

To compute the textual similarity between 2 classes, we used the Rita toolkit. ${ }^{24}$ To find the best trade-off between the 2 objectives (structural and textual measures), we adapted the NSGA-II. ${ }^{23}$ The next section describes the algorithm adaptation to the refactoring problem.

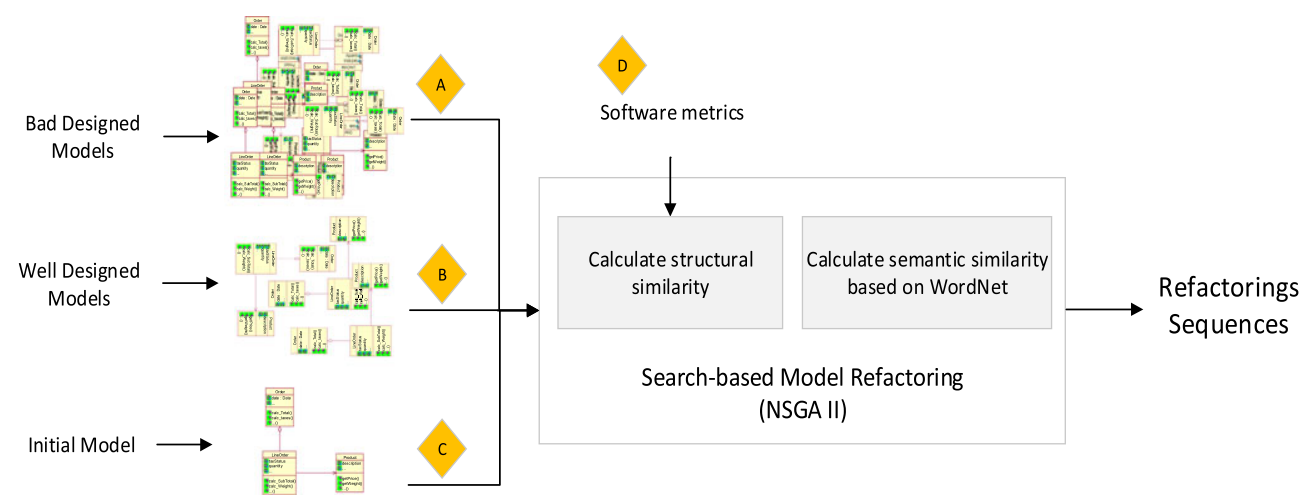

FIGURE 1 Multi-objective model refactoring using examples 
TABLE 1 Considered refactorings in our approach

\begin{tabular}{|c|c|}
\hline Refactoring & Description \\
\hline Extract_Class & Consists of creating a new class and moving the relevant fields and methods from the old class into the new class \\
\hline Rename_Method & $\begin{array}{l}\text { Consists of renaming method with a name that reveals its purpose in order to give more comprehensiveness to the model } \\
\text { design. }\end{array}$ \\
\hline Push_Down_Method & Consists of moving method from a super-class to a specific subclass because it makes sense only there. \\
\hline Push_Down__Attribute & Consists of moving attribute from super class to a specific subclass because it makes sense only there. \\
\hline Rename_Parameter & Consists of renaming parameter within the method parameter list. \\
\hline Move_Attribute & $\begin{array}{l}\text { Consist of moving attribute from a source class to the class destination when it is more used by the second one than the } \\
\text { class on which it is defined. }\end{array}$ \\
\hline Move_Method & $\begin{array}{l}\text { Consists of moving method from a class to another one when it is using or used by more features of the destination class } \\
\text { than the class on which it is defined. }\end{array}$ \\
\hline Pull_Up_Method & $\begin{array}{l}\text { Consists of moving method from some class(es) to the immediate super-class in order to eliminate duplicate methods } \\
\text { among sibling classes, and hence reduce code duplication in general. }\end{array}$ \\
\hline $\begin{array}{l}\text { Replace_Inheritance } \\
\text { With_Delegation }\end{array}$ & $\begin{array}{l}\text { Consists of changing the inheritance relation by a delegation when the subclass uses only part of a super classes interface } \\
\text { or does not want to inherit data. }\end{array}$ \\
\hline
\end{tabular}

TABLE 2 Considered metrics in our approach

\begin{tabular}{|c|c|}
\hline Ref & Metric \\
\hline NA & The number of attributes \\
\hline NPvA & The number of private attributes \\
\hline $\mathrm{NPbA}$ & The number of public attributes \\
\hline NProtA & The number of protected attributes \\
\hline NMeth & The number of methods \\
\hline NPvMeth & The number of private methods \\
\hline NPbMeth & The number of public methods \\
\hline NProtMeth & The number of protected methods \\
\hline NAss & The number of associations \\
\hline NAgg & The number of aggregations \\
\hline NDep & The number of dependencies \\
\hline NGen & The number of generalizations \\
\hline $\mathrm{NAggH}$ & The number of aggregations hierarchies \\
\hline NGenH & The number of generalization hierarchies \\
\hline DIT & «deep inheritance tree»: The longest path from the class to the root of the hierarchy \\
\hline HAgg & «hierarchy aggregation»: The longest path from the class to the leaves. \\
\hline
\end{tabular}

\section{2 | NSGA-II for model refactoring}

\subsection{1 | NSGA overview}

NSGA-II is an evolutionary algorithm based on the non-dominated sorting to solve multi-objective optimization problems. ${ }^{23}$ It was designed to accept an exhaustive list of candidate solutions, which creates a large search space. The idea of NSGA-II consists of finding a representative set of Pareto optimal solutions, called non-dominated solutions. A solution is called non-dominated when no other solution can improve some optimization objective without degrading another. Given a set of objectives $f_{i}, i \in 1, \ldots n$, to maximize, a solution $x$ is said to Pareto dominate another solution $x^{\prime}$ if and only if: ${ }_{i}, f_{i}\left(x^{\prime}\right) \leq f_{i}(x)$ and $\exists j \mid f_{j}\left(x^{\prime}\right)<f_{j}(x)$.

Three main steps characterize the NSGA-II algorithm:

- Create randomly the initial population PO of individuals encoded using a specific representation.

- Create a child population $C O$ generated from the population of parents $P O$ using genetic operators such as crossover and mutation.

- Merge both populations and select a subset of individuals, based on the dominance principle to create the next generation.

This process is repeated until reaching the last iteration according to stopping criteria. 


\subsection{2 | NSGA-II adaptation}

In this section, we describe NSGA-II adaptation to find the best trade-off between structural and textual similarity. As our aim is to maximize structural and textual similarity between a given model (the model to be refactored) and a set of bad designed models in the base of examples (models that have undergone some refactorings) and minimize the structural similarity between a given model and a set of well-designed models in the base of examples (models that do not need any refactoring). We separate each criterion in an objective for NSGA-II. The algorithm takes as input a set of model refactorings' examples (our base of examples), an initial model, and set of metrics. We build an initial population as a set of individuals that stand for possible solutions representing refactorings' sequences possibly applicable to the classes of the initial model (Lines 1-2). An individual is a set of blocks where each block contains a CIM (class chosen from the initial model), a CBE (class chosen from the base of examples) that was matched to CIM, and a list of refactorings which is a subset of the refactorings that were applied to CBE (in its subsequent version) and possibly applicable to the CIM. The next section explains and illustrates the individuals' representation.

Once, a population of refactoring solutions is generated, the main NSGA-II loop (Lines 4-21) consists of evolving a population of candidate solutions towards the best sequence of refactoring, ie, an individual that maximizes as much as possible both the textual and the structural similarities between the classes CIM and CBE that were matched within the individual's blocks. In addition, CIM is compared with all the good examples to identify the closest class in the good model (CGE) using the structural similarity measure. An offspring population $\mathrm{Ct}$ is generated from a parent population Pt using genetic operators (selection, crossover, and mutation) (Line 5) throughout each iteration $t$. After that, Ct and Pt will be merged together to create a global population Gt. Then, each individual I in the population Gt is evaluated using our 2 fitness functions.

After calculating these functions, a list of non-dominated fronts $F(F 1, F 2, \ldots)$ is returned by sorting all the solutions, where $F 1$ represents the set of non-dominated solutions, F2 represents the set of solutions dominated only by solutions in F1, etc (line 11). Then, we build the next population $P t+1$ from the set of non-dominated fronts starting from front $F 1$ to $F i$ (lines 14-17). In general, the number of solutions in all sets from front $F 1$ to $F i$ is larger than the Max_size. To choose exactly Max_size solutions, we sort the solutions of the front Fi using the crowded-comparison operator (< $n$ ) defined $i^{23}$ (line 18). Then, we select the best solutions needed until we reach the Max_size (line 19). The crowded-comparison operator $\left(<_{n}\right)$ is based on non-domination ranking and the crowding distance described in. ${ }^{23}$ Finally, the loop terminates (line 21 ) announcing by that the achievement of termination criterion (ie, maximum iteration number). The result of the algorithm is the set of best solutions, ie, those in the Pareto front of the last iteration (line 22). In the next sub-sections, we give more details concerning the representation of solutions, genetic operators, and the fitness functions.

Algorithm 1. High level pseudo-code for NSGA-Il adaptation to our problem.

\section{Algorithm : NSGA-II search-based model refactoring \\ Input}

Set of bad designed models with refactorings

Set of well-designed models

Initial model

Set of software metrics

Process:

1. I := set (CIM, CBE, CGE, A set of applicable refactorings)

2. $\mathrm{P}_{\mathrm{O}}:=$ set_of $(\mathrm{I})$

3. $t:=0$

4. Repeat

5. $\mathrm{C}_{\mathrm{t}}:=$ apply_Genetic_Operators $\left(\mathrm{P}_{\mathrm{t}}\right)$

6. $\mathrm{G}_{\mathrm{t}}:=\mathrm{P}_{\mathrm{t}} \cup \mathrm{C}_{\mathrm{t}}$

7. For all $\mathrm{I} \in \mathrm{G}_{\mathrm{t}}$

8. SimilarityBE (I) := calculate_Similarity(CIM, CBE)

9. SimilarityGE(I):= calculate_Similarity(CIM, CGE)

10. end For

11. $F:=$ fast_Non_Dominated_Sort $\left(G_{t}\right) / / F=(F 1, F 2, \ldots)$

12. $\mathrm{P}_{\mathrm{t}+1}:=\varnothing$

13. $\mathrm{i}:=1$

14. While $\left|P_{t+1}\right|+\left|F_{i}\right|<$ Max_size

15. $\mathrm{P}_{\mathrm{t}+1}:=\mathrm{P}_{\mathrm{t}+1} \cup \mathrm{F}_{\mathrm{i}}$

16. $\mathrm{i}:=\mathrm{i}+1$

17. end While

18. Sort $\left(F_{\mathrm{i}}\right.$, Crowded_Comparison_Operator)

19. $P_{t+1}:=P_{t+1} \cup F_{i}\left[1\right.$.. (Max_size $\left.\left.-\left|P_{t+1}\right|\right)\right]$

20. $\mathrm{t}:=\mathrm{t}+1$ 
21. Until $\mathrm{t}=$ Max_iteration

22. best_solutions := first_front $\left(P_{t}\right)$

Output :

best_solutions.

\section{INDIVIDUAL REPRESENTATION}

To apply NSGA-II, we represent a candidate solution (ie, an individual) as a set of blocks. A block is a quadruplet of (CIM, CBE, CGE, Applicable refactorings to $\mathrm{CIM}$ ), where CIM is a class chosen from the initial model, $\mathrm{CBE}$ is a class chosen from the base of examples that was matched to CIM, CGE is a class chosen from the well-designed model, and finally the list of refactorings which is a subset of the refactorings that were applied to $\mathrm{CBE}$ (in its subsequent versions and that can be applied to CIM). Figure 2 shows an example of an individual (solution). The refactoring selection process adopted within a block considers some constraints in order to avoid conflicts and inconsistencies. For example, if the CIM class does not have any attribute or method and the CBE class requires a Move_Attribute or Add_Parameter refactoring operation, then we discard these refactoring operations because we cannot apply them to the CIM class.

The bottom part of Figure 3 shows an example of a candidate solution (ie, an individual) composed of 3 blocks. Each block contains 1 refactoring operation. Therefore, the individual represents a refactorings' sequence to apply and the CIM's on which they apply. The top part of Figure 3 shows the fragments of an initial model before and after the suggested refactorings' sequence by the individual (at the bottom of the figure) were applied. Notice that the same refactoring operation could be included several times in the same individual.

The generation of the initial population requires a maximum individual size as parameter. This parameter can be chosen randomly or specified the user. In our implementation, we adopted the first choice in order to obtain individuals with different sizes. After that, we assign randomly for each individual: (1) A set of CIM, (2) their matched CBE's, and (3) the sub-set of refactorings possibly applicable to the CIM among the refactorings proposed by the CBE.

\section{SELECTION AND GENETIC OPERATORS}

a. Selection

There are many methods how to select the best individuals that will participate in the next generation (eg, roulette wheel selection, Boltzmann selection, tournament selection, rank selection, steady state selection, etc.). NSGA-II uses binary tournament selection ${ }^{23}$ to derive a child population $Q_{t}$ (ie, the set of individuals that will undergo the crossover and mutation operators) from a parent population $P_{t}$. The binary tournament selection involves running several "tournaments". Each tournament involves 2 randomly selected individuals from the population. This will give all individuals of the population the chance to be selected and preserves diversity.

\begin{tabular}{|c|}
\hline CIM \\
\hline CBE \\
\hline CGE \\
\hline Applicable refactorings to CIM \\
\hline
\end{tabular}

FIGURE 2 Block representation

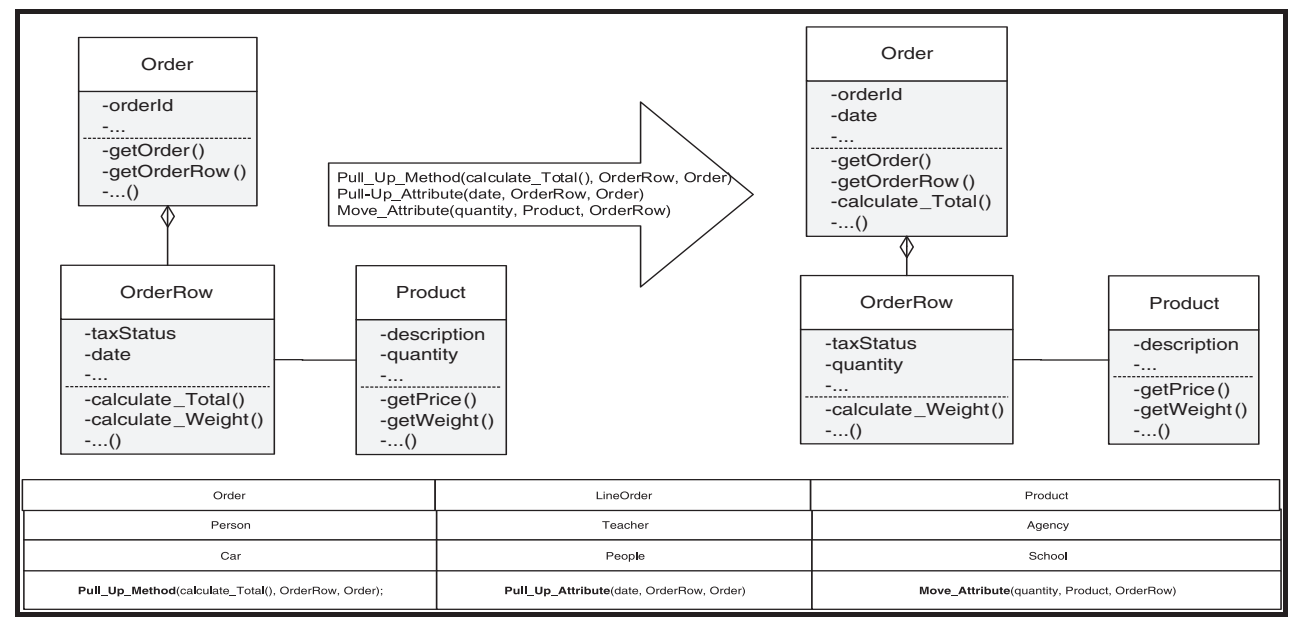

FIGURE 3 Individual representation 


\begin{tabular}{|c|c|c|c|c|c|c|c|}
\hline \multicolumn{8}{|c|}{ Cross Point } \\
\hline \multirow{4}{*}{$\mathrm{P} 1$} & Order & OrderRow & Product & \multirow{4}{*}{ P'1: } & School & Pilot & Product \\
\hline & Person & Teacher & Agency & & Course & Student & Agency \\
\hline & Car & People & School & & College & Bill & School \\
\hline & 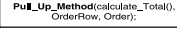 & 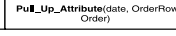 & 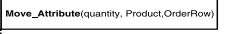 & & 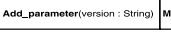 & 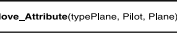 & 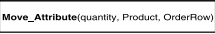 \\
\hline \multirow{4}{*}{ P2: } & School & Pilot & & \multirow{4}{*}{$P^{\prime} 2:$} & Order & OrderRow & \\
\hline & Course & Student & & & Person & Teacher & \\
\hline & College & Bill & & & Car & People & \\
\hline & Add_parameter(version : String) & Move_AAtributotypepePlane, Pilot, Plane & & & 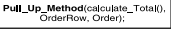 & 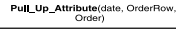 & \\
\hline
\end{tabular}

FIGURE 4 Crossover operator

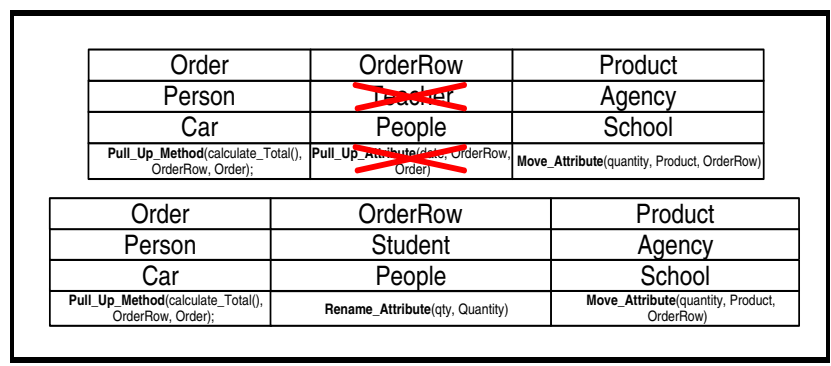

FIGURE 5 Mutation operator

b. Crossover

We use a simple, random, cut-point crossover. For each crossover, 2 individuals are selected by applying the tournament selection. ${ }^{23}$ The crossover happens only with a certain probability. The crossover operator allows creating 2 offsprings $\mathrm{P}_{1}{ }^{\prime}$ and $\mathrm{P}_{2}{ }^{\prime}$ from the 2 selected parents $P_{1}$ and $P_{2}$. Figure 4 illustrates a 1-point crossover in which 1 point is selected from the parents $P_{1}$ and $P_{2}$. Everything before the cross point is swapped between the parents, producing 2 children $\mathrm{P}_{1}{ }^{\prime}$ and $\mathrm{P}_{2}{ }^{\prime}$.

c. Mutation

The mutation operator consists of randomly changing 1 or more components of the list representing an individual (solution). Given a selected individual, the mutation operator first randomly selects 1 or more block of the sequence corresponding to the individual, and then the CBE of each block will be replaced by another one chosen randomly from the base of examples. Figure 5 illustrates the effect of a mutation operation. The CBE (Teacher and its refactorings list) of the second block is replaced by the CBE Student and its refactorings taking into consideration the constraints mentioned before.

For all the above change operators, we used a repair operator that eliminates redundant, conflicting, or infeasible refactorings. The repair operator can either delete or randomly change these dimensions with new refactoring types or change the controlling parameter of these refactorings. Of course, these issues are also penalized by the fitness function when the solution becomes infeasible, and it will increase the distance with design examples.

\section{MULTI-CRITERIA EVALUATION OF INDIVIDUALS}

In practice, we should formalize the evaluation of an individual as a mathematical function called "fitness function". Two different fitness functions have been considered in this work: (1) the first one calculates the structural similarity between CIM and CBE and between CIM and CGE; (2) the second one calculates the textual similarity between CIM and CBE. The idea behind is that a candidate solution that displays high structural and textual similarities between CIM and CBE should give the best sequence of refactorings.

a. Structural criterion

The structural criterion is evaluated using the fitness function denoted by Structural_Similarity by formula 1 and 2.

$$
\text { Structural_Similarity }(C M I, C B E)=\frac{1}{m} \sum_{i=1}^{m} \operatorname{Sim}\left(C M I_{i}, C B E_{i}\right)
$$




$$
\operatorname{Sim}\left(C M l_{i}, C B E_{i}\right)=\left\{\begin{array}{cc}
1 & \text { if } C M l_{i}=C B E_{i} \\
0 \quad \text { if }\left(C M l_{i}=0\right. & \text { and } \left.C B E_{i} \neq 0\right) \text { or }\left(C M l_{i} \neq 0 \text { and } C B E_{i}=0\right) \\
\frac{C M l_{i}}{C B E_{i}} & \text { if } C M l_{i}<C B E_{i} \\
\frac{C B E_{i}}{C M l_{i}} & \text { if } C B E_{i}<C M l_{i}
\end{array}\right.
$$

Where $m$ represents the number of metrics. $C \mathrm{CM}_{\mathrm{i}}, \mathrm{CBE}_{\mathrm{i}}$ represent the $\mathrm{i}^{\text {th }}$ metric value of the $\mathrm{CIM}$ and $\mathrm{i}^{\text {th }}$ metric value of the $\mathrm{CBE}$, respectively. Thus, we define the structural fitness function of a solution, normalized in the range $[0,1]$, as:

$$
f_{\text {Structural }}=\frac{1}{n} \sum_{j=1}^{n} \text { Structural_Similarity }\left(C M I_{B j}, C B E_{B j}\right)
$$

Where $n$ represents the individual size (number of blocks), $C M I_{B j}$ and $C_{B E}$ represent the classes in the first 2 parts of the $j^{\text {th }}$ block of the individual. To illustrate the computation of the structural fitness function, we considered a system containing 2 classes as shown in Table 3 and a base of examples containing 2 classes shown in Table 4. In this example, we use 6 metrics given for each class in the model in Table 3 and each class of the base of examples in Table 4.

We present 2 individuals $I_{1}$ and $I_{2}$, respectively, composed by 2 blocks (Plane/Store and Person/Car) and 1 block (Person/Strore). The fitness function calculated on these solutions has the value:

$$
\begin{gathered}
f_{\text {Structural } I_{1}=} \frac{1}{2}\left[\frac{1}{6}\left[\left(\frac{3}{5}+1+1+0+\frac{2}{4}+0\right)+\left(\frac{3}{6}+\frac{2}{3}+\frac{5}{7}+1+1+1\right)\right]\right]=0,66 \\
f_{\text {Structural } I_{2}}=\left[\frac{1}{6}\left[\left(\frac{3}{3}+\frac{2}{3}+\frac{4}{7}+0+\frac{2}{4}+0\right)\right]\right]=0,46
\end{gathered}
$$

In this case, the evaluation process will choose the individual having the maximum value of fitness function, then $I_{1}$ will be chosen as best individual.

\section{b. Design consistency criterion}

To formulate the textual similarity fitness function, we used Rita toolkit ${ }^{24}$ which enables to compute the degree of likeness between 2 concepts based on their meaning. Specifically, we used this tool to calculate the textual similarity distance between 2 classes using their names. Thus, the textual similarity between 2 classes Class1 and Class2, denoted as Textual_Similarity(Class1, Class2), corresponds to the textual similarity distance between Class1's name and Class2's name. The idea behind consist of breaking down the 2 class names, if the class name is a complex name, into different words. To this end, we used predefined functions in Rita toolkit that allow us the extraction of nouns, verbs, adverbs, etc. Then, we calculate the distance between different obtained combinations. Finally, the design

TABLE 3 Classes from the initial model and their metrics values

\begin{tabular}{lllllll} 
CIM & NPvA & NPbA & NPbMeth & NPvMeth & NAss & NGen \\
\hline Plane & 5 & 2 & 4 & 2 & 2 & 2 \\
Person & 3 & 3 & 7 & 0 & 2 & 0 \\
\hline
\end{tabular}

TABLE 4 Classes from the base of examples and their metrics values

\begin{tabular}{lllllll} 
CBE & NPvA & NPbA & NPbMeth & NPvMeth & NAss & NGen \\
\hline Store & 3 & 2 & 4 & 0 & 4 & 0 \\
Car & 6 & 2 & 5 & 0 & 2 & 0 \\
\hline
\end{tabular}


consistency fitness function of a solution corresponds to the average of distances of all the blocks of the solution as shown by the formula 4 .

$$
f_{\text {Textual }}=\frac{1}{n} \sum_{j=1}^{n} \text { Textual_Similarity }\left(C M I_{B j}, C B E_{B j}\right)
$$

Where $\mathrm{n}$ is the number of blocks in the solution and $\mathrm{CMI}_{\mathrm{Bj}}$ and $\mathrm{CBE}_{\mathrm{Bj}}$ are the classes composing the first 2 parts of the $\mathrm{j}^{\text {th }}$ block of the solution. We considered the textual similarity when the model is compared with the bad designed models because we adapted the refactorings applied to that bad designed model to our context. Thus, the textual similarity is important along with the structural one. However, the dissimilarity with the well-designed model is used as a helper objective just to identify refactoring opportunities but not to the correction of these identified defects. The structural function is used by both fitness functions and the semantic function is just used by part the first fitness function that calculates the similarity between the model to refactor and bad examples (average of structural and textual similarities).

\section{I EXPERIMENTATIONS WITH THE APPROACH}

This section describes the evaluation steps of our approach. It starts by presenting our supporting tools. Then, we define our research questions. Finally, we describe our experimental settings, and we present and discuss the results of the experimentations.

\section{1 | Supporting tools}

Three preliminary steps are needed for the validation of our approach: (1) we implemented a parser to analyze Java source code and generate a predicate model as illustrated in Figure 6; (2) we run the parser on 8 Java open source projects (Ant, JabRef, JGraphx, JHotDraw, GanttProject, JRDF, Xerces, and Xom) in order to obtain their predicate models; and (3) we completed the obtained models in the second step by manually entering the refactoring operations extracted with Ref-Finder ${ }^{26}$ that these projects have undergone. Figure 7 illustrates an example of a CBE. The RefFinder tool permits detection of 68 refactorings that include a set of atomic refactorings by using logic-based rules executed by a logic-programming engine. Ref-Finder helps finding refactorings that a system has undergone by comparing different versions of the system. Two reasons were behind the use of Ref-finder tool: (1) build the base of examples and (2) compute the precision and recall of our approach. We also used another tool called Rita toolkit ${ }^{24}$ to calculate the textual similarity.

A plugin was developed to support our approach using Eclipse ${ }^{\mathrm{TM}}$ development environment. Figure 8 shows a screenshot of the model refactoring plugin perspective. The plugin supports many heuristic-based algorithms for refactoring and hence enable to enter many controlling parameters depending on the chosen algorithm. For the NSGA-II refactoring algorithm, it takes as input a base of examples of models and their related refactorings, an initial model to refactor, and a set of metrics. The user also specifies the population size, the number of iterations, and

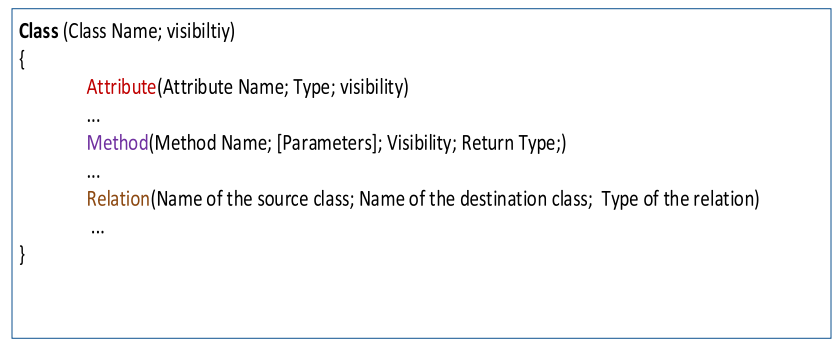

FIGURE 6 Class representation in the generated model

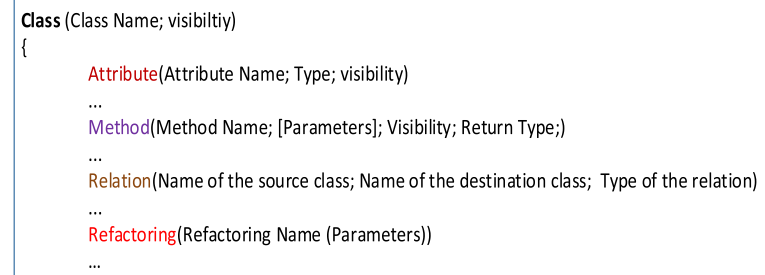

FIGURE 7 A class completed with its subsequent refactorings 
the solution size (we also can keep this value randomly). It generates as output a Pareto-front which contains optimal solutions of sequence of refactorings to be applied on the analyzed system.

\section{2 | Experimental setup}

Several tests are done to set the NSGA-II parameters. The stopping criterion was set to 1000 iterations, the Max_size of the population to 30 , the crossover probability to 0.9 , and the mutation probability to 0.4 . We obtained these values by trial and error. In our case, we selected a high mutation rate because it allows the continuous diversification of the population, which discourages premature convergence to occur. A standard desktop computer (i7 CPU running at $3 \mathrm{GHz}$ with 8GB of RAM) was used to run the NSGA-II algorithm. The run-time of our algorithm with a 1000 iterations (stopping criteria) was less than 4 minutes, which indicates the scalability of our approach from the performance standpoint. However, the run-time depends on the number of refactorings and the size of the models in the base of examples.

We analyzed 8 open-source Java projects to answer the research questions reported above:

- Ant (v1.8.4): A Java library that is mainly used for building Java applications. Ant provides support to compile, assemble, test, and run Java applications.

- GanttProject (v2.0.10): A Java project that supports project management and scheduling.

- JabRef (v2.7): A graphical application for managing bibliographical databases.

- JGraphx (v1.10.4.0): A Java Swing diagramming (graph visualization) library.

- JHotDraw (v5.2): A framework for the creation of drawing editors.

- JRDF (v0.5.6.2): A Java library for parsing, storing, and manipulating RDF (Resource Description Framework).

- Xerces (v2.7): A set of parsers compatible with Extensible Markup Language (XML).

- Xom (v1.2.8): A new XML object model.

The choice of these open source projects is based on the fact that they are medium-sized open-source projects, and most of them were analyzed in related work (eg, $\left.{ }^{18,26-28}\right)$. Most of these open source projects have been actively developed over the past 10 years. Table 5 provides some relevant information about these projects. Table 6 describes the number of good and bad examples and their sizes extracted from Ant 1.8.4,

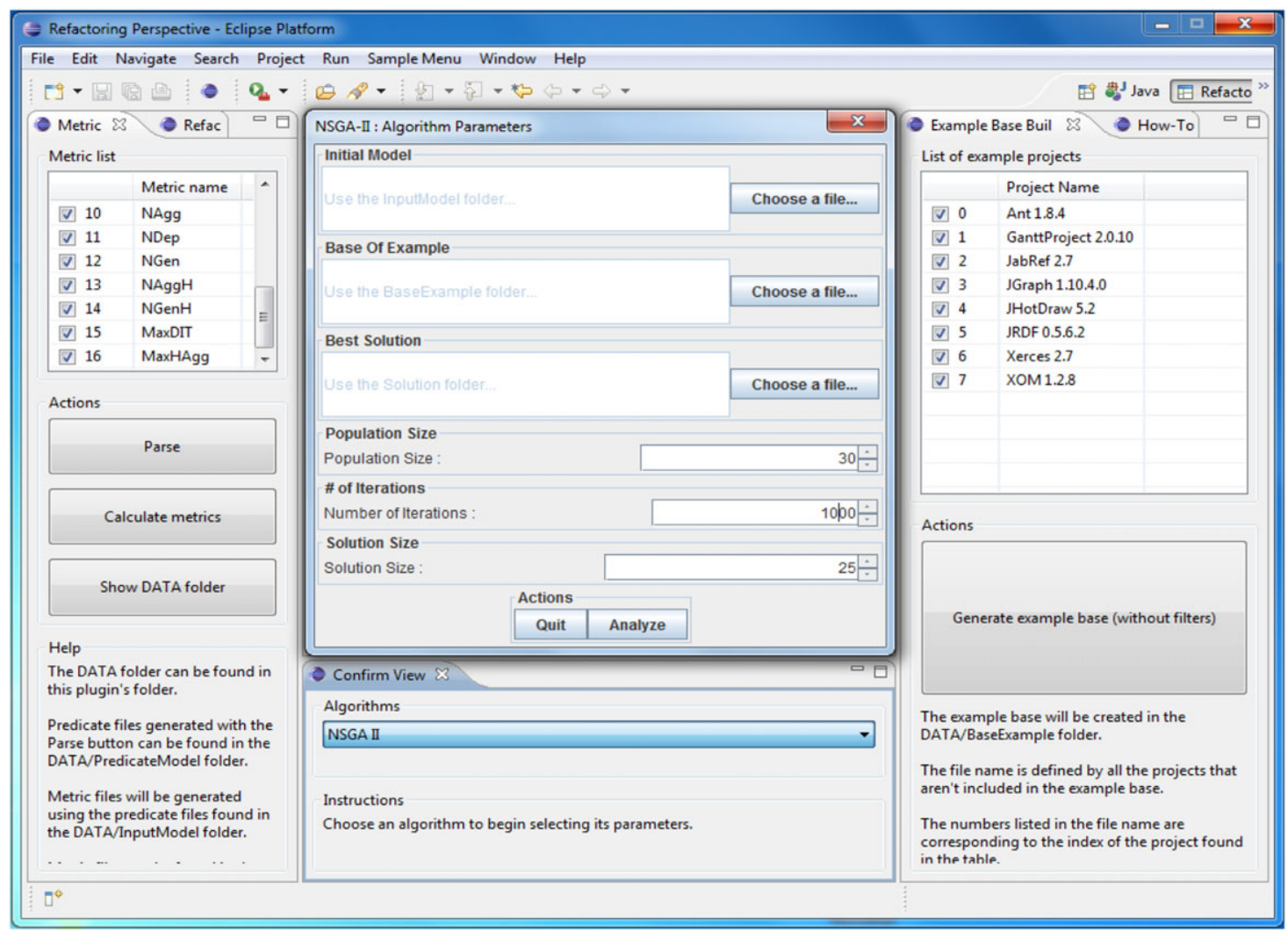

FIGURE 8 Model refactoring plugin 
GanttProject 2.0.10, JHotDraw 5.2, and Xerces 2.7. Most of the examples are selected based on the previous studies that analyzed these systems. ${ }^{29-31}$

\section{3 | Measures of precision and recall}

To answer our research questions, we used 2 measures: Precision and Recall. These measures stem originally from the area of information retrieval (IR). Precision is given by Equation 5. It is equal to the ratio of the "Number of correct refactorings detected" to the "Total number of refactorings detected". A recall is given by Equation 6. It is equal to the ratio of the "Number of correct refactorings detected" to the "Number of correct refactorings". Both values may range from $0 \%$ to $100 \%$, whereas a higher value is better than a lower one. We consider that the threshold for the correctness of recommended refactorings is $85 \%$.

$$
\begin{gathered}
\text { PRECISION }=\frac{\text { Number of correct refactorings detected }}{\text { Total number of refactorings detected }} \\
\text { RECALL }=\frac{\text { Number of correct refactorings detected }}{\text { Number of correct refactorings }}
\end{gathered}
$$

\subsection{Results and discussion}

Our results are presented based on 2 indicators: precision and recall. For our validation, we conducted multiple executions (40 executions) of our approach on the all considered projects. Figure 9 illustrates the average of precision and recall values for each open source project over the 40 executions. Figure 10 illustrates the box plots of the precision scores of our algorithm on the different projects based on 40 executions. Similarly, Figure 11 illustrates the box plots of the precision scores of our algorithm on the different projects based on 40 executions.

We noticed very high values of the average of precision and recall, which is over $90 \%$ for GanttProject, JHotDraw, JRDF, and Xerces and less than $90 \%$ for the rest of projects (Ant, JabRef, JGraphx [88\%] and Xom [86\%]) under test. Indeed, the most common intervals (80\%-100\%) displayed by Figures 10 and 11 prove that precision and recall scores are approximately the same for different executions in the 8 projects under analysis. The most common range of $20 \%$ between the minimum (80\%) and the maximum (100\%) for each project is a sign of stability of the approach. In fact, the minimum values of the recall on the different systems based on the different runs were between $\mathbf{7 9 . 8 \%}$ and $\mathbf{8 0 . 1 \%}$. The skewed distributions between the results of different projects are mainly related to the used training examples as input (different programming contexts, etc.). For example, for JHotDraw and GanttProject, the average of the precision values is around $90 \%$ and is over than $94 \%$ for the average of the recall, while Xerces had approximately the same average of the precision and recall around $92 \%$. These results allow us to positively answer our first research question RQ1 and conclude that the obtained results are very encouraging.

\begin{tabular}{|c|c|c|c|c|}
\hline Model & Classes & Methods & Attributes & Expected refactorings \\
\hline Ant 1.8.4 & 824 & 2090 & 1048 & 139 \\
\hline GanttProject 2.0.10 & 479 & 960 & 495 & 91 \\
\hline JabRef 2.7 & 594 & 253 & 237 & 32 \\
\hline JGraphx 1.10.4.0 & 191 & 1284 & 420 & 96 \\
\hline JHotDraw 5.2 & 160 & 519 & 141 & 71 \\
\hline$J R D F$ v0.5.6.2 & 734 & 19 & 10 & 41 \\
\hline Xerces 2.7 & 625 & 2113 & 1408 & 182 \\
\hline Xom 1.2.8 & 252 & 186 & 31 & 36 \\
\hline
\end{tabular}

TABLE 5 Case study settings

TABLE 6 Statistics about the extracted sets of bad and good design examples

\begin{tabular}{lll} 
Model & $\begin{array}{l}\text { \#bad examples } \\
\text { (min\#classes, max\#classes) }\end{array}$ & $\begin{array}{l}\text { \#good examples } \\
\text { (min\#classes, max\#classes) }\end{array}$ \\
\hline Ant 1.8.4 & $72(8,23)$ & $42(7,29)$ \\
\hline GanttProject 2.0.10 & $49(6,21)$ \\
JHotDraw 5.2 & $34(9,19)$ \\
Xerces 2.7 & $38(6,14)$ & 19(8, 24) \\
\hline
\end{tabular}




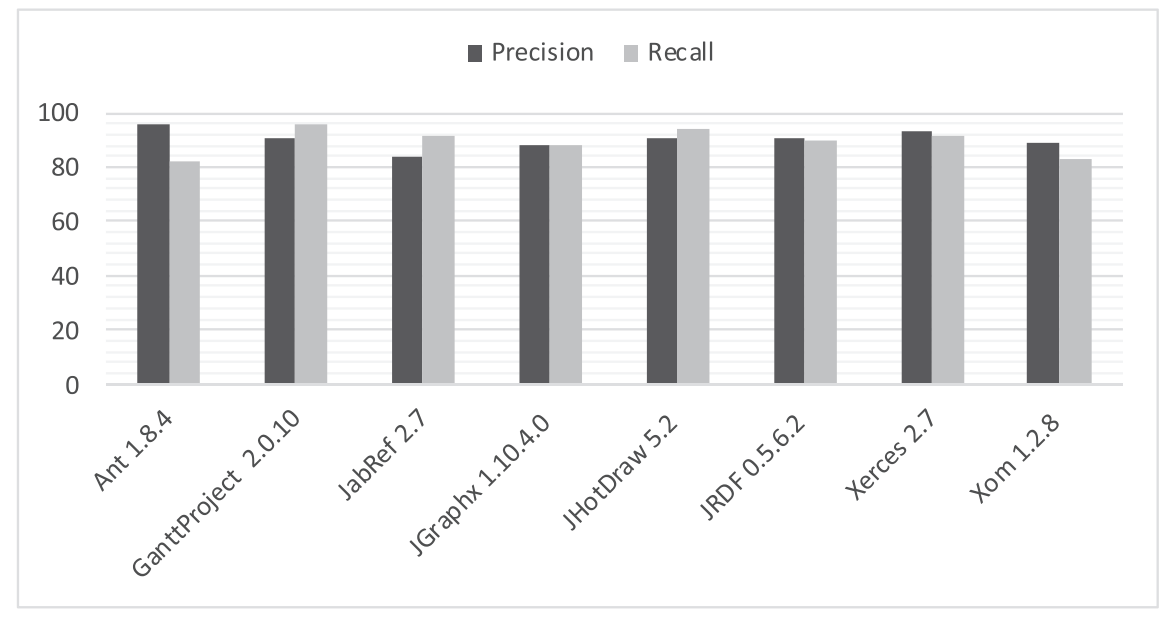

FIGURE 9 Average of precision and recall over 40 execution of our approach on all projects

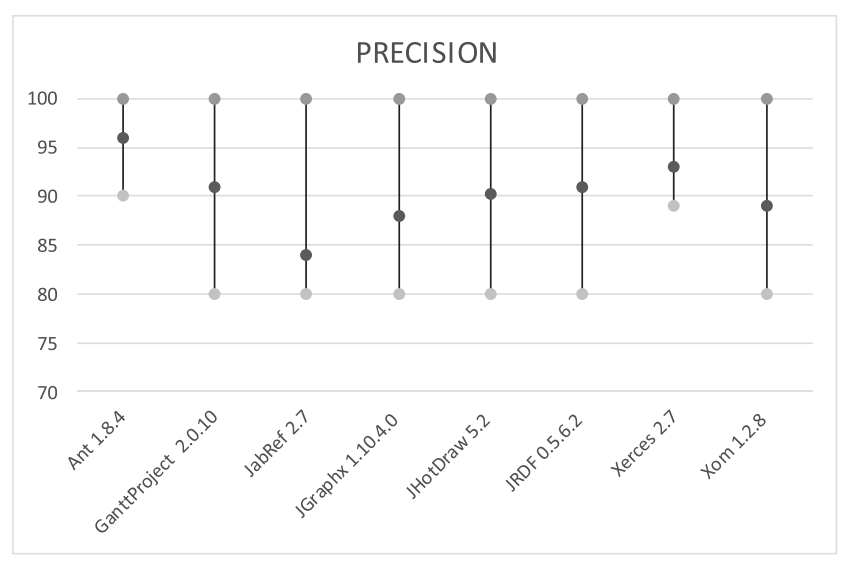

FIGURE 10 The minimum, maximum, and average of the precision scores of our algorithm on the different projects based on 40 executions

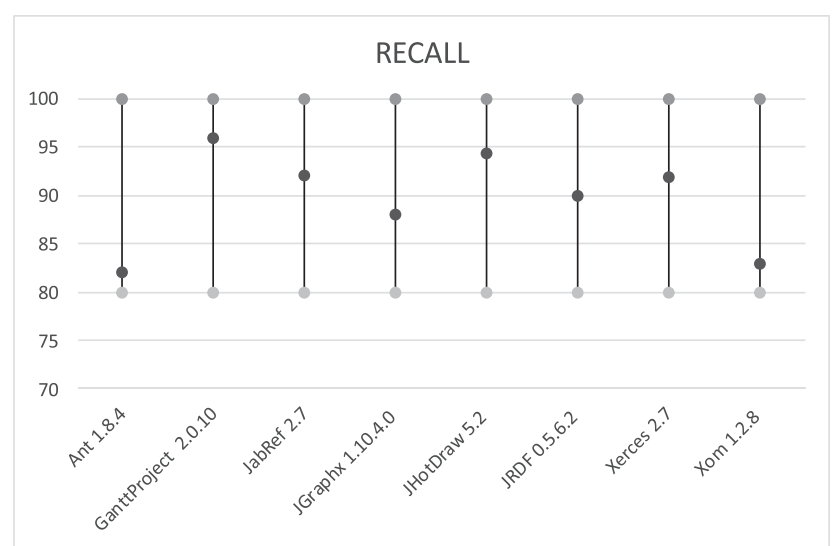

FIGURE 11 The minimum, maximum, and average of the recall scores of our algorithm on the different projects based on 40 executions

The advantage with the multi-objective approach is that NSGA-II, unlike GA, produces a set of solutions called the Pareto front. In our context, NSGA-II converges to Pareto-optimal solutions that are considered as good trade-off between structural and textual similarities. Figure 12 display the Pareto-front for NSGA-II obtained on the considered open source projects. In these figures, each point is a solution with the structural similarity score represented in the $\mathrm{x}$-axis, the textual similarity score in the $\mathrm{y}$-axis. The best solutions exist in the corner representing the Pareto front that maximizes the values of the textual and the structural similarities.

To answer the second research question, RQ2, we compared our NSGA-II adaptation to the current, state-of-the-art refactoring approaches widely used multi-objective algorithm, MOPSO, ${ }^{21}$, using the same adapted fitness function, NSGA-II without the use of good design examples 


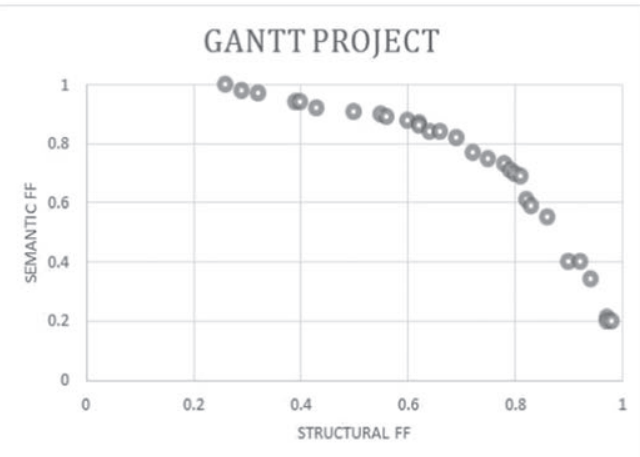

(A)

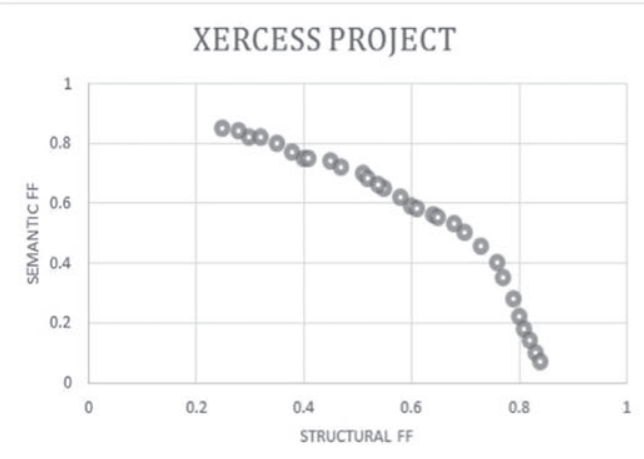

(C)

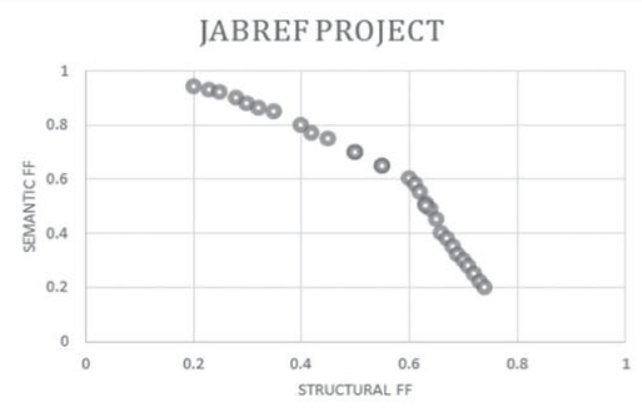

(E)

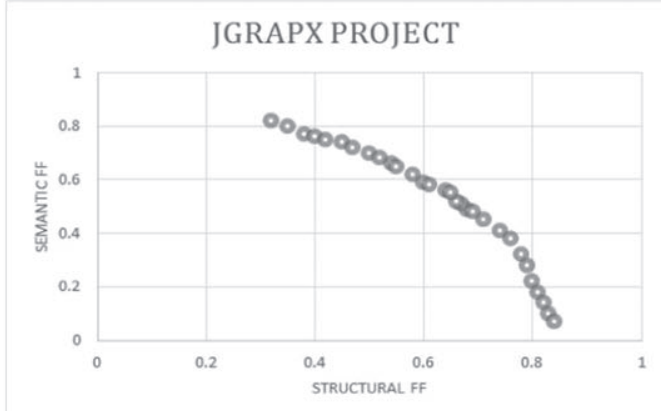

(G)

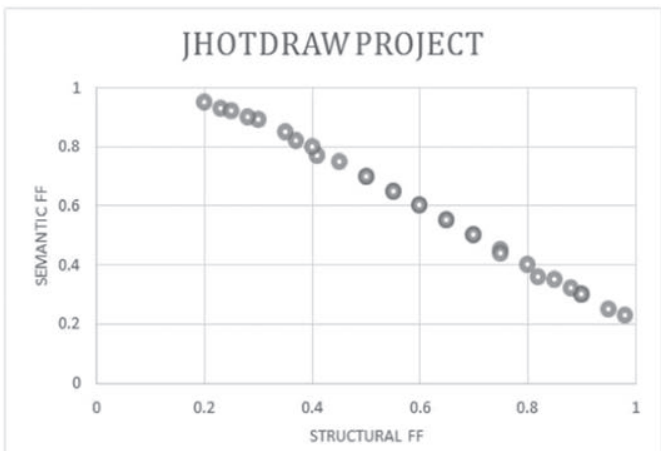

(B)

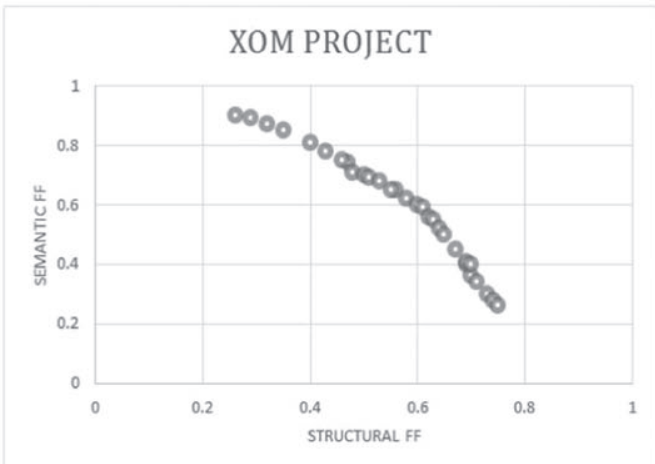

(D)

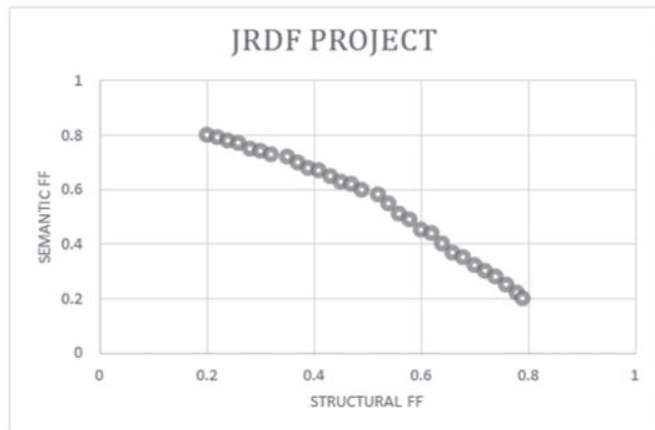

(F)

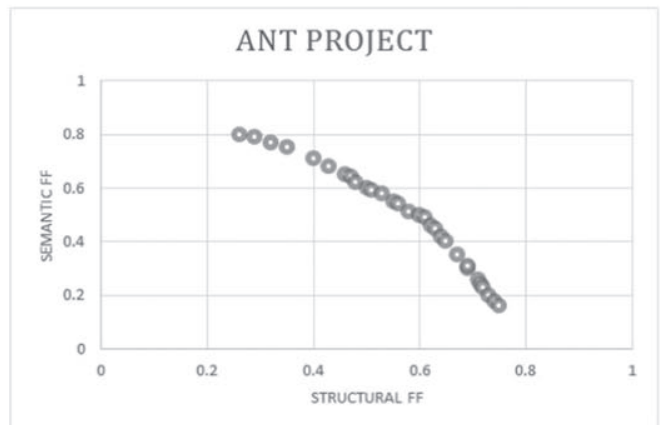

(H)

FIGURE 12 Pareto front for NSGA-II obtained on 8 open source systems: (A) GanttProject, (B) JHotDraw, (C) Xercess, (D) Xom, (E) JabRef, (F) JRDF, (G) JGraphx, and (H) Ant

(NSGA-II BE), ${ }^{20}$ to a mono objective approach (MONO) using GA, to our previous work (MOREX: MOdel Refactoring by EXamples), ${ }^{17}$ to a genetic programming (GP) based approach ${ }^{24}$ and to a random search approach. In MONO, we defined the fitness function as combination of both structural and textual fitness functions. The MOPSO used in this paper is the Non-dominated Sorting PSO (NSPSO) proposed by Li. ${ }^{21}$ In a random search, the change operators (crossover and mutations) are not used, and populations are generated randomly and evaluated using the 2 fitness functions. To better evaluate the relevance of considering well-designed model fragment examples, we compared our NSGA-II adaptation to our previous work based also on NSGA-II but without the use of good examples (called NSGA-II BE). ${ }^{20}$ In MOREX, ${ }^{17}$ we used a single-objective 
GA to propose refactorings, ie, we considered only 1 fitness function based on the structural similarity which is a combination of software metrics. In $\mathrm{GP}^{22}$ the authors proposed an approach to generate detection rules based on quality metrics by using GP.

It is clear from Figures 13 and 14 that our NSGA-Il adaptation outperforms all the mono-objective approaches (MONO, MOREX, and GP) in $100 \%$ of experiments. As shown by Figures 13 and 14, NSGA-II had higher average of precision and recall than MONO, MOREX, and GP, and it beats by far the random search approach. For example for GanttProject, the NSGA-II average precision and average recall values are $91 \%$ and $96 \%$, respectively, while these values are $82 \%$ and $86 \%$ in MOREX, $78 \%$ and $82 \%$ in GP, and $41 \%$ and $43 \%$ in the random search algorithm where these values do not exceed $50 \%$ for all the 8 projects. For example, we noticed an increase of $9 \%$ on average between NSGA-II and MOREX that could be considered as great improvement. This improvement can be explained by the fact that NSGA-II aims to find a compromise between 2 similarities (structural and textual). However, MOREX did not consider textual similarity but only structural one. When comparing NSGA-II with the remaining approaches, we considered the best solution selected from the Pareto-optimal front using the knee point-based strategy. ${ }^{32}$ The results obtained in Figures 13 and 14 support the claim that our NSGA-II formulation provides a good trade-off between structural and textual similarities and outperforms on average the MOPSO approach, except for Ant, JRDF, and XOM opens source systems where precision and recall are quite similar.

As the considered algorithms are meta-heuristics, they can produce different results on every run when applied to the same problem instance. For this reason, we used the P-values of the Wilcoxon rank-sum test ${ }^{33}$ as a statistical test to compare the results of the 5 algorithms: NSGA-II, MONO, MOREX, ${ }^{17} \mathrm{NSGA}-I I \mathrm{BE},{ }^{20} \mathrm{GP},{ }^{22}$ and MOPSO. ${ }^{21}$ We independently performed 40 executions using the 6 algorithms in MONO, MOREX, ${ }^{17}$ $\mathrm{GP},{ }^{22} \mathrm{NSGA}-\mathrm{II} \mathrm{BE},{ }^{20}$ and MOPSO, ${ }^{21}$ for the 8 open-source projects that we used in our experiment. In our context, a $P$-value that is less than or equal to a $(=0.05)$ means that the distributions of the results of the 5 algorithms are different in a statistically significant way. In fact, we computed the $P$-values of MONO, MOREX, GP, and MOPSO results compared with NSGA-II. In this way, we could decide whether the outperformance of our approach over the MONO, MOREX ${ }^{17} \mathrm{GP}$, NSGA-II BE, ${ }^{20}$ and MOPSO ${ }^{21}$ approach is statistically significant. Tables 7 and 8 display the precision and recall median values of NSGA-II, MONO, MOREX, GP, NSGA-II BE, ${ }^{20}$ and MOPSO ${ }^{21}$ for the 8 open source projects, respectively. For example, for Ant project, the $P$-value for the precision median results of MONO compared with NSGA-II is 0.0103 while the $P$-value of the recall median results of MONO compared with NSGA-II is 0.0113. The P-value for the precision median results of MOREX compared with NSGA-II is 0.0224, while the $P$-value of the recall median results of MOREX compared with NSGA-II is 0.0213 . The P-value for the precision median GP compared with NSGA-II is 0.0112 , while the $P$-value of the recall median results of GP compared with NSGA-II is 0.0142 . In addition, the $P$-value for the precision median results of MOPSO compared with NSGA-II is 0.0098 , while the P-value of the recall median results of MOPSO compared with NSGA-II is 0.0087 .
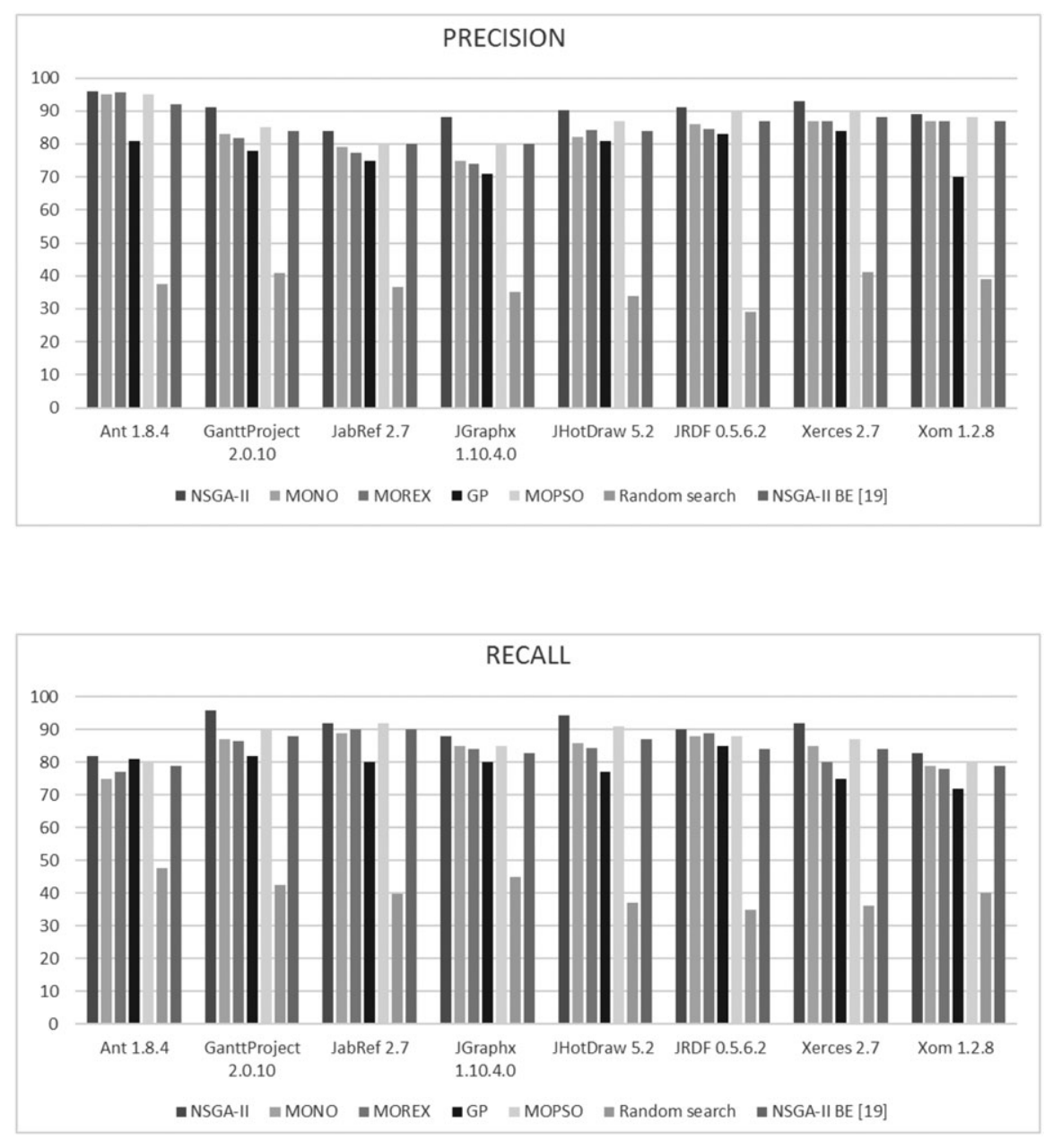

FIGURE 13 Comparison between NSGA-II, MONO, MOREX, ${ }^{16} \mathrm{GP},{ }^{21} \mathrm{MOPSO},{ }^{20}$ NSGA-II $\mathrm{BE},{ }^{19}$ and random search in terms of precision
FIGURE 14 Comparison between NSGA-II, MONO, MOREX, ${ }^{16} \mathrm{GP},{ }^{21}$ MOPSO, ${ }^{20}$ NSGA-II $\mathrm{BE},{ }^{19}$ and random search in terms of recall 
TABLE 7 Precision median values of NSGA-II, MONO, MOREX, ${ }^{16} \mathrm{GP}^{21}$ NSGA-II BE, ${ }^{19}$ and MOPSO ${ }^{20}$ over 40 independent simulation runs

\begin{tabular}{|c|c|c|c|c|c|c|c|c|c|c|c|}
\hline \multirow[b]{2}{*}{ Model } & \multicolumn{6}{|c|}{ Precision (\%) } & \multicolumn{5}{|c|}{$P$-value $(\leq 0.05)$} \\
\hline & $\begin{array}{l}\text { NSGA- } \\
\text { II }\end{array}$ & $\begin{array}{l}\text { NSGA-II } \\
\mathrm{BE}^{19}\end{array}$ & MONO & MOREX & GP & MOPSO & $\begin{array}{l}\text { NSGA-II vs } \\
\text { MONO }\end{array}$ & $\begin{array}{l}\text { NSGA-II } \\
\text { vs }^{19} \\
\end{array}$ & $\begin{array}{l}\text { NSGA-II vs } \\
\text { MOREX }\end{array}$ & $\begin{array}{l}\text { NSGA-II vs } \\
\text { GP }\end{array}$ & $\begin{array}{l}\text { NSGA-II vs } \\
\text { MOPSO }\end{array}$ \\
\hline Gantt & 90 & 84 & 83 & 84 & 80 & 75 & 0.0015 & 0.0014 & 0.0158 & 0.0101 & 0.0012 \\
\hline JabRef & 84 & 80 & 79 & 78 & 77 & 73 & 0.0101 & 0.0111 & 0.0124 & 0.0077 & 0.0045 \\
\hline JGraphx & 86 & 80 & 75 & 77 & 73 & 70 & 0.0017 & 0.0047 & 0.0212 & 0.0111 & 0.0014 \\
\hline JRDF & 90 & 87 & 86 & 88 & 84 & 82 & 0.0028 & 0.0027 & 0.0189 & 0.0045 & 0.0084 \\
\hline Xerces & 92 & 88 & 87 & 89 & 86 & 83 & 0.0102 & 0.0043 & 0.0211 & 0.0059 & 0.0047 \\
\hline Xom & 87 & 87 & 84 & 87 & 86 & 70 & 0.0094 & 0.0052 & 0.0051 & 0.0091 & 0.0031 \\
\hline
\end{tabular}

TABLE 8 Recall median values of NSGA-II, MONO, MOREX, ${ }^{16} \mathrm{GP},{ }^{21} \mathrm{NSGA}-\mathrm{II} \mathrm{BE},{ }^{19}$ and MOPSO ${ }^{20}$ over 40 independent simulation runs

\begin{tabular}{|c|c|c|c|c|c|c|c|c|c|c|c|}
\hline \multirow[b]{2}{*}{ Model } & \multicolumn{6}{|c|}{ Recall (\%) } & \multicolumn{5}{|c|}{$P$-value $(\leq 0.05)$} \\
\hline & $\begin{array}{l}\text { NSGA- } \\
\text { II }\end{array}$ & $\begin{array}{l}\text { NSGA-II } \\
\mathrm{BE}^{19}\end{array}$ & MONO & MOREX & GP & MOPSO & $\begin{array}{l}\text { NSGA-II vs } \\
\text { MONO }\end{array}$ & $\begin{array}{l}\text { NSGA-II } \\
\text { vs }^{19} \\
\end{array}$ & $\begin{array}{l}\text { NSGA-II vs } \\
\text { MOREX }\end{array}$ & $\begin{array}{l}\text { NSGA-II vs } \\
\text { GP }\end{array}$ & $\begin{array}{l}\text { NSGA-II vs } \\
\text { MOPSO }\end{array}$ \\
\hline Ant & 81 & 79 & 73 & 78 & 77 & 80 & 0.0113 & 0.0026 & 0.0213 & 0.0142 & 0.0087 \\
\hline Gantt & 94 & 88 & 86 & 88 & 86 & 81 & 0.0011 & 0.0134 & 0.0211 & 0.0091 & 0.0071 \\
\hline JabRef & 91 & 90 & 88 & 90 & 90 & 78 & 0.0009 & 0.0087 & 0.0016 & 0.0075 & 0.0061 \\
\hline JGraphx & 87 & 83 & 83 & 84 & 83 & 79 & 0.0102 & 0.0231 & 0.0058 & 0.0097 & 0.0004 \\
\hline JRDF & 89 & 84 & 86 & 86 & 88 & 83 & 0.0087 & 0.0011 & 0.0198 & 0.0117 & 0.0076 \\
\hline Xerces & 91 & 84 & 84 & 85 & 78 & 74 & 0.0101 & 0.0340 & 0.0029 & 0.0131 & 0.0044 \\
\hline Xom & 82 & 79 & 77 & 77 & 75 & 69 & 0.0105 & 0.0014 & 0.0158 & 0.0141 & 0.0059 \\
\hline
\end{tabular}

Accordingly, we infer that the precision and recall median values of our algorithm are statistically different from the MONO, MOREX, GP, NSGA-II $\mathrm{BE},{ }^{20}$ and the MOPSO ones on each of the systems based on the fact that these $P$-values are less than a $(=0.05)$. We consequently conclude that our approach is more effective than these 4 approaches, and specifically it is more effective than an approach based only on the structural similarity without taking into account the context of the entities of analyzed models (ie, MOREX). This observation allows us to positively answer our second research question RQ2.

The proposed approach is different than a classical data mining technique or a manual inspection of the history to identify similar refactoring patterns. First, the proposed approach does not find similarities between refactorings, but the current model design is compared with examples of bad and good design models then the applied refactorings to that similar bad design model are adapted to the current context. It will be difficult for a developer to identify similarities between model fragments based on the history of changes, especially when the number of changes is high. Second, our multi-objective approach does not require using examples from previous releases of the project to evaluate. The experiments show that examples from open source systems could be a very good starting point for developers in practice/industry to use our technique. Finally, our approach generates a set of Pareto front solutions and not a single solution, which is the case of machine learning and data mining algorithms. Thus, the developer can select a solution based on his preferences and not limited to 1 set of recommended refactorings.

Because we viewed the matching problem as a combinatorial problem addressed with heuristic search, it is important to contrast the correctness results with the execution time. We executed our algorithm on a standard desktop computer (Pentium CPU running at $3 \mathrm{GHz}$ with 8GB of RAM). The average execution time is shown in Figure 15. The execution time of the mono-objective algorithms is slightly lower than our NSGA-II approach and NSGA-II BE, ${ }^{20}$ but the MOPSO one was higher than ours (due to the used changes operators by MOPSO). In any case, our approach is meant to apply to situations where the execution time is not the primary concern. Figure 16 shows the distribution of the different types of refactoring recommended by our approach. It is clear that move method, extract class, and push down method are the most frequent refactorings between the different solutions.

\section{4 | THREATS TO VALIDITY}

We consider the use of the Ref_finder tool to build the base of examples, and at the same time, we compare the results obtained by our algorithm to those given by Ref_finder as threats to the generalization of our approach. In general, we do not need a big number of examples to obtain good results, which confirm the reliability of the proposed approach. We showed, in our experiment, that some open source projects used out of the box could produce good refactoring results for the systems under analysis. However, we agree that, sometimes, within specific contexts, it is difficult to 


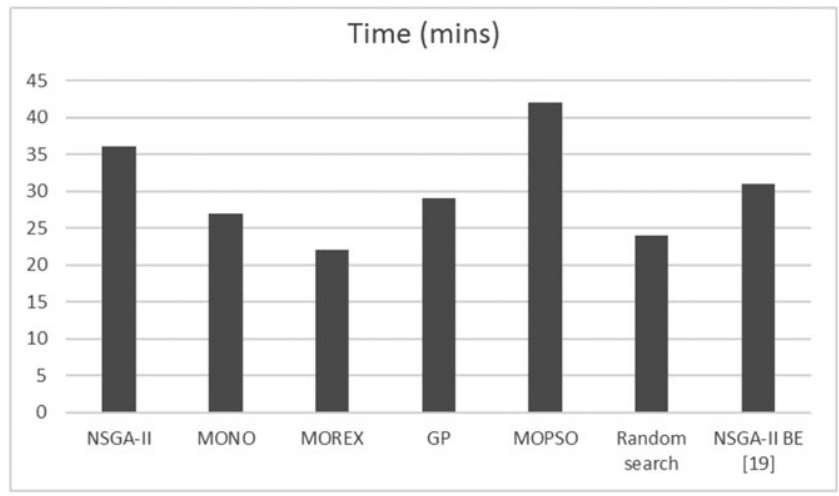

FIGURE 15 Comparison between NSGA-II, MONO, MOREX, $,{ }^{16} \mathrm{GP},{ }^{21} \mathrm{NSGA}-\mathrm{II} \mathrm{BE},{ }^{19} \mathrm{MOPSO},{ }^{20}$ and random search in terms of average execution time on the different systems

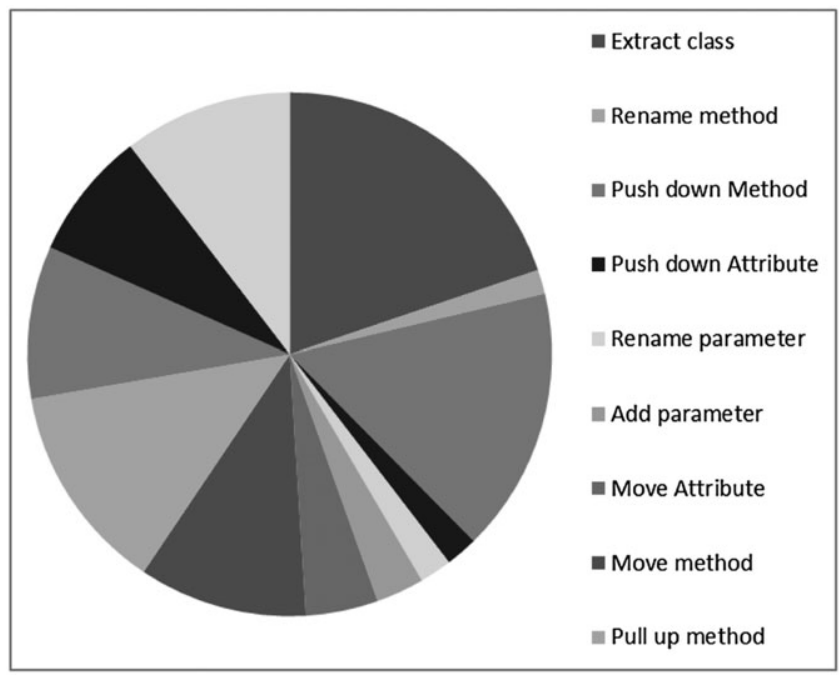

FIGURE 16 The average distribution of refactoring types in the best solutions recommended by our approach on the different models

define and find refactorings' opportunities. Applications from different domains may have dissimilar design practices, and developers may have different opinions on how to define a good and bad design example. In fact, the quality of our results heavily depends on the examples introduced as inputs. In an industrial setting, we could expect a company to start with some few open source projects and gradually migrate its set of refactoring examples to include context-specific data. In fact, the definition of "good" and "bad" examples is left to the developers based on their preferences and best/bad design practices. A possible use of previous releases of evolved models could be a good strategy to adapt in order to define a set of bad and good design fragments. This might be essential if we consider that different languages and software infrastructures have different best/worst practices. Another threat is related to the used textual measures. Sometimes, 2 classes have similar names, but they are semantically equivalent. Also, 2 classes might have different names but be semantically similar.

\section{5 | RELATED WORK}

In this section, we classified a relevant existing work that tackled the automation of refactoring activities using search-based techniques into 2 main groups: single-objective and multi-objective optimization approaches.

In the first group, most of existing works defined their fitness function based on software metrics in order to find the best sequence of refactorings (eg, ${ }^{15,22,34-44}$ ). A comparative study of 4 heuristic techniques applied to the refactoring problem was presented by O'Keeffe et al ${ }^{34}$ using a fitness function that combine 11 metrics to evaluate the quality improvements. They experiment the 4 techniques on 5 open-source systems. They find that hill-climbing outperforms the other 3 algorithms. Another single-objective optimization was proposed by Seng et al ${ }^{15}$ using on the GA in order to suggest a list of refactorings. The single fitness function used in this proposal aims to maximize a weighted sum of a set of metrics to improve the class structure of a software system. These metrics are mainly related to the coupling, cohesion, complexity, and stability. The authors have defined some refactorings' preconditions able to preserve the behavior but not the semantics domain of the software program. However, authors have validated their proposal only on the move method refactoring. The refactoring schedule problem was considered by Qayum et $\mathrm{al}^{35}$ as a graph 
transformation problem expressing as a search for an optimal path. To do that, they used Ant colony optimization in the graph where nodes and edges represent, respectively, refactoring candidates and dependencies between them. However, the domain semantics of the software system and its runtime behavior were not considered. Last but not least, Kessentini et $\mathrm{al}^{22}$ have proposed a single-objective optimization approach using GA to find the best sequence of refactoring operations at code level aiming to improve its quality by minimizing the design defects' number detected in the source code.

In the second group, Pareto-front concept has been used by Harman et $\mathrm{al}^{19}$ in order to improve search based refactoring approaches. The authors have combined 2 software metrics: (1) CBO (coupling between objects) and (2) SDMPC (standard deviation of methods per class). Each metric has been assigned to 1 objective (fitness function). The authors found that the proposed multi-objective algorithm is able to find a good sequence of move method refactorings. The obtained sequence represents the best trade-off between CBO and SDMPC to improve code quality. Another multi-objective optimization approach has been proposed by Ouni et al ${ }^{18}$ using NSGA-II providing the best trade-off between 2 objectives: (1) quality based on the metric that calculates the number of corrected defects detected in the initial system and (2) effort based on the hat calculates the code modifications score metric. In another work, Ouni et al ${ }^{45}$ tried to find the best trade-off to find the best sequence of refactorings by maximizing the quality improvements and minimizing semantic errors. Ó Cinnéide et al ${ }^{46}$ have investigated, via an empirical study, about the assessment of some structural software metrics and the relationships between them, based on a variety of search techniques (Paretooptimal search, semi-random search). In conclusion, vast majority of existing work on search-based software engineering approaches focused only on the program structure improvements based on a set of software metrics in both single and multi-objectives approaches. Thaina et al ${ }^{47}$ proposed a recent systematic literature review on search-based refactoring.

Interactive techniques have been generally introduced in the literature of Search-Based Software Engineering and especially in the area of software modularization. Hall et $\left.\right|^{48}$ treated software modularization as a constraint satisfaction problem. The idea of this work is to provide a baseline distribution of software elements using good design principles (eg, minimal coupling and maximal cohesion) that will be refined by a set of corrections introduced interactively by the designer. The approach, called SUMO (Supervised Re-modularization), consists of iteratively feeding domain knowledge into the remodularization process. The process is performed by the designer in terms of constraints that can be introduced to refine the current modularizations. Initially, the system begins with generating a module dependency graph from an input system. This dependency is based on the correlation between software elements (coupling between methods, shared attributes etc.). Possible modularizations are then generated from the graph using multiple simulated authoritative decompositions. Then, using a clustering technique called Bunch, an initial set of clusters is generated that serves as an input to SUMO.

Bavota et $\mathrm{al}^{49}$ presented the adoption of single objective interactive GAs in software re-modularization process. The main idea is to incorporate the user in the evaluation of the generated re-modularizations. Interactive GAs (IGAs) extend the classic GAs by partially or entirely involving the user in the determination of the solution's fitness function. The basic idea of the IGA is to periodically add a constraint to the GA such that some specific components shall be put in a given cluster among those created so far. Initially, the IGA evolves similarly to the non-interactive GA. After a userdefined set of iterations, the individual with the highest fitness value is selected from the population set (in the case of single-objective GA) or from the first front (in the case of multi-objective GA) and presented to the user. After analyzing the current modularization, the user provides feedback in terms of constraints dictating for example, that a specific element needs to be in the same cluster as another one. Overall, the above existing studies of interactive re-modularization are limited to few types of refactoring such as moving classes between packages and splitting packages. Furthermore, the interaction mechanism is based on the manual evaluation of proposed re-modularization solutions, which could be a time-consuming process. A recent study ${ }^{50}$ extended our previous work ${ }^{51}$ to propose an interactive search-based approach for refactoring recommendations. The developers have to specify a desired design at the architecture level then the proposed approach try to find the relevant refactorings that can generate a similar design to the expected one.

On the other hand, there are some contributions that focused on the automation of refactoring activities at the model level based on rules. In general, the used rules have been expressed either as assertions (ie, invariants, pre-and post-condition), ${ }^{52,53}$ or as graph transformations targeting refactoring operations in general (eg, ${ }^{54,55}$ ) or refactorings related to design patterns' applications (eg, ${ }^{7}$ ). For example, Ragnhild et $\mathrm{al}^{52}$ have proposed to detect some parts of the model that need refactoring using declarative rules. However, this proposal requires an important number of rules. In addition, refactoring rules must be complete, consistent, non-redundant, and correct to specify clearly and completely of the refactorings. Refactoring rules have been used also by ElBoussaidi and Mili ${ }^{7}$ in order to specify design patterns' applications. To do that, the authors represent the design problems (solved by these patterns) based on models. Then, the obtained models will be transformed using refactoring rules according to the solutions proposed by the patterns. However, models are not able to represent all design problems at hand. For example, the problem space for the observer pattern is quite large, and the problem cannot be captured in a single, or a handful of problem models. ${ }^{7}$ In conclusion, a common issue in most of the proposed approaches is how to sequence and compose the refactoring rules.

Some other studies have tackled the refactoring opportunities detection at model level using code smells defined as bad design choices which could negatively impact some code qualities such as maintainability, changeability, and comprehensibility. ${ }^{56}$ Indeed, during the evolution of the system, code-smells can emerge and then represent patterns of software design. These patterns can be a source of problems in the further development and maintenance of the system. Fowler et $\mathrm{al}^{2}$ identified and defined 22 Code Smells aiming to indicate software refactoring opportunities and "give you indications that there is trouble that can be solved by a refactoring". Zhang et al ${ }^{57}$ investigated about code-smells that need more attention than any other Van Emden and Moonen ${ }^{58}$ and Mantyla ${ }^{59}$ proposed 2 approaches that aim to detect and analyze code smells for java programs. Previous empirical studies have analyzed the impact of code-smells on different software maintainability factors including 
defects $^{60}$ and effort. ${ }^{61}$ In fact, software metrics (quality indicators) are sometimes difficult to interpret and suggest some actions (refactoring) as noted by Anda et $\mathrm{al}^{62}$ and Marinescu et al. $^{63}$

\section{6 | CONCLUSION}

We presented a by example search-based approach that exploits both structural and design consistency information to improve the automation of suggesting refactoring. It takes as input a model to be refactored, a base of examples of models and their subsequent refactorings, and a list of metrics and textual measures calculated on both the initial model and the models in the base of examples. The output is a solution to the refactoring problem. A solution is a sequence of refactoring operations that should be applied to the initial model and that displays the best compromise between the 2 criteria: structural and textual similarities. In contrast to existing work on refactorings, the design consistency is a major concern in our paper.

Our experimentation shows that our technique outperforms state-of-the-art techniques where single-objective and multi-objective is used. We evaluated our approach on real-world models, and the obtained results indicate that the proposed refactorings are comparable to those expected. We also tested the stability of our approach by performing multiple executions on the projects at hand and checking the stability of precision and recall values over the multiple executions. These results allowed us to conclude that the proposed approach is more efficient and promising than approaches that do not consider the design consistency in their optimization objectives.

In the future, we plan to extend our base of examples to include more refactoring operations. We also plan to analyze the domain-specific impact on the obtained results.

\section{ACKNOWLEDGEMENT}

This work was made possible by the NSF Award \#1661422 and NPRP grant \# [7-662-2-247] from Qatar Research Fund (a member of Qatar Foundation). The findings achieved herein are solely the responsibility of the authors.

\section{ORCID}

Adnane Ghannem (D) http://orcid.org/0000-0002-1320-899X

Mohammad Salah Hamdi (D) http://orcid.org/0000-0001-7420-3036

Ghizlane El Boussaidi (D) http://orcid.org/0000-0001-6145-774X

\section{REFERENCES}

1. ISO/IEC, International Standard-ISO/IEC 14764 IEEE Std 14764-2006 Software Engineering; Software Life Cycle Processes \&; Maintenance. ISO/IEC 14764:2006 (E) IEEE Std 14764-2006 Revision of IEEE Std 1219-1998), 2006: p. 01-46.

2. Fowler M. Refactoring: Improving the Design of Existing Code. Boston, MA, USA: Addison-Wesley; 1999.

3. Mens T, Tourwé T. A survey of software refactoring. IEEE Trans Softw Eng. 2004;30(2):126-139.

4. Opdyke FW. Refactoring : A program restructuring aid in designing object-oriented application frameworks. 1992, University of Illinois at UrbanaChampaign.

5. Moha N. DECOR : détection et correction des défauts dans les systèmes orientés objet. 2008, Université de Montréal \& Université des Sciences et Technologies de Lille: Montréal. p. 157-179.

6. Du Bois B, Demeyer S, Verelst J. Refactoring-improving coupling and cohesion of existing code. In: Proceedings of the 11th Working Conference on Reverse Engineering (WCRE). Delft University of Technology, Netherlands: IEEE Computer Society; 2004.

7. El-Boussaidi G, Mili H. Understanding design patterns - what is the problem? Softw. Pract. Exper. 2011;42:1495-1529.

8. Mens T, Taentzer G, Dirk M. Challenges in Model Refactoring. in Proceedings of the 1st Workshop on Refactoring Tools (WRT). Germany: University of Berlin; 2007.

9. Zhang J, Lin Y, Gray J. Generic and Domain-Specific Model Refactoring Using a Model Transformation Engine. In: Beydeda S, Book M, Gruhn V, eds. Model-Driven Software Development. Berlin, Heidelberg: Springer; 2005.

10. Brown JW. AntiPatterns: Refactoring software, architectures, and projects in crisis. WILEY ed. 1998:336.

11. Douglas CS. Guest editor's introduction: Model-driven engineering. Comput. J. 2006;39(2):41-47.

12. El-Boussaidi G, Mili H. Detecting patterns of poor design solutions using constraint propagation. In: Proceedings of the 11 th international conference on Model Driven Engineering Languages and Systems. Toulouse, France: Springer-Verlag; 2008:189-203.

13. Kessentini M, Sahraoui H, Boukadoum M, Wimmer M. Search-based design defects detection by example. In: Proceedings of the 14th international conference on Fundamental approaches to software engineering: Part of the joint European conferences on theory and practice of software. Saarbrücken, Germany: Springer-Verlag; 2011.

14. O' Keeffe M, O' Cinneide M. Search-based software maintenance. In: Proceedings of the 10th European Conference on Software Maintenance and Reengineering (CSMR). Bari, Italy; 2006.

15. Seng O, Stammel J, Burkhart D. Search-based determination of refactorings for improving the class structure of object-oriented systems. In: Proceedings of the 8th Annual Conference on Genetic and Evolutionary Computation. Seattle, Washington, USA: ACM; 2006.

16. Kessentini M, Sahraoui H, Boukadoum M. Model transformation as an optimization problem. In: Proceedings of the 11th International Conference on Model Driven Engineering Languages and Systems. Toulouse, France: Springer-Verlag; 2008. 
17. Ghannem A, El-Boussaidi G, Kessentini M. Model refactoring using examples: A search-based approach. J Softw Evol Proc. 2014;26:692-713.

18. Ouni A, Kessentini M, Sahraoui H, Boukadoum M. Maintainability defects detection and correction: A multi-objective approach. Journal of Automated Software Engineering, JASE. 2013;20(1):47-79.

19. Harman M, Tratt L. Pareto optimal search based refactoring at the design level. In: Proceedings of the 9th Annual Conference on Genetic and Evolutionary Computation (GECCO). London, England, UK: ACM; 2007:1106-1113.

20. Ghannem A, ElBoussaidi G, Kessentini M. Example-based model refactoring using multi-objective optimization. In: North American Search Based Software Engineering Symposium. Michigan, Detroit, USA; 2015:104-127.

21. Deb K, Pratap A, Agarwal S, Meyarivan T. A fast and elitist multiobjective genetic algorithm: NSGA-II. IEEE Transactions on Evolutionary Computation. 2002;6(2):182-197.

22. Li X. A non-dominated sorting particle swarm optimizer for multiobjective optimization. In: Cantú-Paz E et al., eds. Genetic and Evolutionary Computation - GECCO 2003. Berlin Heidelberg: Springer; 2003:37-48.

23. Kessentini M, Kessentini W, Sahraoui H, Boukadoum M, Ouni A. Design defects detection and correction by example. In: Proceedings of the 19th International Conference on Program Comprehension (ICPC). Kingston, ON, CANADA: IEEE Computer Society; 2011:81-90.

24. Howe CD. RiTa: Creativity support for computational literature. In: Proceedings of the 7th ACM Conference on Creativity and Cognition. Berkeley, CA, USA: ACM; 2009:205-210.

25. Genero M, Piattini M, Calero C. Empirical validation of class diagram metrics. in Proceedings of the International Symposium in Empirical Software Engineering (ISESE). 2002. Nara, Japan.

26. Miryung K, Matthew G, Alex L, Napol R. Ref-Finder: A refactoring reconstruction tool based on logic query templates. In: Proceedings of the 18th ACM SIGSOFT International Symposium on Foundations of Software Engineering. Santa Fe, New Mexico, USA: ACM; 2010:371-372.

27. Moha N, Yann-Gael G, Duchien L, Le Meur AF. DECOR: A method for the specification and detection of code and design smells. IEEE Trans. Softw. Eng. 2010;36(1):20-36.

28. Ghannem A, Kessentini M, El-Boussaidi G. Detecting model refactoring opportunities using heuristic search. In: Litoiu M, Stroulia E, MacKay S, eds. Proceedings of the 2011 Conference of the Center for Advanced Studies on Collaborative Research (CASCON). Riverton, NJ, USA: IBM Corp; 2011.

29. Kessentini M, Vaucher S, Sahraoui HA. Deviance from perfection is a better criterion than closeness to evil when identifying risky code. ASE. 2010;113-122.

30. Kessentini M, Kessentini W, Sahraoui HA, Boukadoum M, Ouni A. Design defects detection and correction by example. ICPC. 2011;81-90.

31. Mansoor U, Kessentini M, Wimmer M, Deb K. Multi-view refactoring of class and activity diagrams using a multi-objective evolutionary algorithm. Software Quality Journal. 1-29. to appear

32. Rachmawati L, Srinivasan D. Multiobjective evolutionary algorithm with controllable focus on the knees of the Pareto front. IEEE Trans. Evol. Comput. 2009;13(4):810-824.

33. Wilcoxon F. Individual comparisons by ranking methods. In: Kotz S, Johnson N, eds. Breakthroughs in Statistics. New York: Springer; 1992:196-202.

34. O' Keeffe M, O' Cinnéide M. Search-based refactoring: An empirical study. J. Softw. Evol. Process. 2008;20(5):345-364.

35. Qayum F, Heckel R. Local search-based refactoring as graph transformation. In: Proceedings of the 1st International Symposium on Search Based Software Engineering (SSBSE). Springer Berlin Heidelberg: Cumberland Lodge, Windsor, UK; 2009.

36. ben Fadhel A, Kessentini M, Langer P, Wimmer M. 2012, September. Search-based detection of high-level model changes. In Software Maintenance (ICSM), 2012 28th IEEE International Conference on (pp. 212-221). IEEE.

37. Ghannem A, El-Boussaidi G, Kessentini M. Model refactoring using interactive genetic algorithm. In: Proceedings of the 5th Symposium on Search Based Software Engineering (SSBSE). Berlin Heidelberg: Springer; 2013.

38. Bechikh S, Kessentini M, Said LB, Ghédira K. Chapter four-preference incorporation in evolutionary multiobjective optimization: A survey of the stateof-the-art. Adv Comput. 2015;98:141-207.

39. Kalboussi S, Bechikh S, Kessentini M, Said LB. Preference-based many-objective evolutionary testing generates harder test cases for autonomous agents. In: International Symposium on Search Based Software Engineering. Berlin, Heidelberg: Springer; 2013:245-250.

40. Kessentini M, Bouchoucha A, Sahraoui H, Boukadoum M. (2010). Example-based sequence diagrams to colored petri nets transformation using heuristic Search. Modelling Foundations and Applications, 156-172.

41. Kessentini M, Langer P, Wimmer M. "Searching models, modeling search: On the synergies of SBSE and MDE." Proceedings of the 1st International Workshop on Combining Modelling and Search-Based Software Engineering. IEEE Press, 2013.

42. Rapu D, et al. Using history information to improve design flaws detection. in Software Maintenance and Reengineering, 2004. CSMR 2004. Proceedings. Eighth European Conference on. 2004.

43. Yamashita A, Moonen L. Do code smells reflect important maintainability aspects? In 28th IEEE International Conference on Software Maintenance (ICSM). 2012.

44. Ouni A, Kessentini M, Sahraoui H. "Search-based refactoring using recorded code changes." Software Maintenance and Reengineering (CSMR), 2013 17th European Conference on. IEEE, 2013.

45. Ouni A, Kessentini M, Sahraoui H, Hamdi MS. Search-based refactoring: Towards semantics preservation. On 28th IEEE International Conference in Software Maintenance (ICSM), 2012. https://doi.org/10.1109/ICSM.2012.6405292 2012

46. O' Cinnéide M, Tratt L, Harman M, Counsell S, Hemati MI. Experimental assessment of software metrics using automated refactoring. In: Proceedings of the ACM-IEEE International Symposium on Empirical Software Engineering and Measurement. Lund, Sweden: ACM; 2012.

47. Mariani T, Vergilio SR. A systematic review on search-based refactoring. Inf Softw Technol. 2017;83, C (March 2017):14-34. https://doi.org/10.1016/j. infsof.2016.11.009

48. Hall M, Walkinshaw N, McMinn P. 'Supervised software modularization. 28th IEEE International Conference on Software Maintenance. pp. 23-30.' (2012. 2012) 
49. Bavota G, Carnevale F. 'Putting the developer in a loop: An interactive GA for software re-modularization. Search based software engineering, Lecture notes in computer science volume 7515. pp 75-89' (2012. 2012)

50. Lin Y, Peng X, Cai Y, Dig D, Zheng D, Zhao W. 'Interactive and guided architectural refactoring with search-based recommendation', International Symposium on the Foundations of Software Engineering (FSE 2016), Accepted, 2016

51. Mkaouer MW, Kessentini M, Bechikh S, Deb K, Ó Cinnéide M. 'Recommendation system for software refactoring using innovization and interactive dynamic optimization'. ASE 2014:331-336

52. Ragnhild VDS, Jonckers V, Mens T. A formal approach to model refactoring and model refinement. SoSyM. 2007;6(2):139-162.

53. Marc Van Kempen M, Chaudron M, Kourie D, Boake A. Towards proving preservation of behaviour of refactoring of UML models. In: Proceedings of the 2005 annual research conference of the South African institute of computer scientists and information technologists on IT research in developing countries (SAICSIT); 2005. South African Institute for Computer Scientists and Information Technologists, Republic of South Africa.

54. Mens T, Taentzer G, Runge O. Analysing refactoring dependencies using graph transformation. Software and Systems Modeling. 2007;6(3):269-285.

55. Biermann E. EMF model transformation based on graph transformation: Formal foundation and tool environment. In: Proceedings of the 5th International Conference on Graph Transformations (ICGT). Enschede, The Netherlands: Springer-Verlag; 2010.

56. Brito e Abreu, F, Melo W. Evaluating the impact of object-oriented design on software quality. In: Proceedings of the 3rd International Software Metrics Symposium, 1996.

57. Zhang M, Hall T, Baddoo N. Code Bad Smells: A review of current knowledge. J Softw Evol Proc. 2011;23(3):179-202.

58. Van Emden E, Moonen L. Java quality assurance by detecting code smells. In: Proceedings of the Ninth Working Conference on Reverse Engineering. 2002.

59. Mäntylä MV, Lassenius C. Subjective evaluation of software evolvability using code smells: An empirical study. C Empir Software Eng. 2006;11(3):395431.

60. Monden A, Nakae D, Kamiya T, Sato S, Matsumoto K. Software quality analysis by code clones in industrial legacy software. In: the Proceedings of the Eighth IEEE Symposium on Software Metrics; 2002.

61. Deligiannis I, Shepperd M, Roumeliotis M, Stamelosd I. An empirical investigation of an object-oriented design heuristic for maintainability. J Syst Softw. 2003;65(2):127-139.

62. Anda B. Assessing software system maintainability using structural measures and expert assessments. in Software Maintenance, 2007. ICSM 2007. IEEE International Conference on. 2007.

63. Marinescu R. Detection strategies: metrics-based rules for detecting design flaws. In: Proceedings of the 20th IEEE International Conference on Software Maintenance, 2004.

How to cite this article: Ghannem A, Kessentini M, Hamdi MS, El Boussaidi G. Model refactoring by example: A multi-objective search based software engineering approach. J Softw Evol Proc. 2018;30:e1916. https://doi.org/10.1002/smr.1916 